\title{
The Complexity of Degree Anonymization by Vertex Addition ${ }^{\text {负 }}$
}

\author{
Robert Bredereck ${ }^{1}$, Vincent Froese ${ }^{2}$, Sepp Hartung, André Nichterlein, \\ Rolf Niedermeier, Nimrod Talmon ${ }^{3}$ \\ Institut für Softwaretechnik und Theoretische Informatik, TU Berlin, Germany
}

\begin{abstract}
Motivated by applications in privacy-preserving data publishing, we study the problem of making an undirected graph $k$-anonymous by adding few vertices (together with some incident edges). That is, after adding these "dummy vertices", for every vertex degree $d$ appearing in the resulting graph, there shall be at least $k$ vertices with degree $d$. We explore three variants of vertex addition (justified by real-world considerations) and study their (parameterized) computational complexity. We derive mostly intractability results, even for very restricted cases (including trees and bounded-degree graphs) but also obtain some encouraging fixed-parameter tractability results.
\end{abstract}

Keywords: graph modification, degree-constrained editing, NP-hardness, parameterized complexity, W-hardness, fixed-parameter tractability, kernelization

\section{Introduction}

This work is concerned with making an undirected graph $k$-anonymous, that is, transforming it (at "low cost") into a graph where every vertex degree occurs either zero or at least $k$ times. This graph modification scenario is motivated by data privacy requests in social networks; it focuses on degree-based attacks on identity disclosure of network nodes. Liu and Terzi [26] (also see Clarkson et al.

\footnotetext{
${ }^{2}$ A preliminary version of this work has been presented at the 2014 International Conference on Algorithmic Aspects of Information and Management (AAIM 2014), Vancouver, Canada, July 2014 [5].

Email addresses: robert.bredereck@tu-berlin.de (Robert Bredereck), vincent.froese@tu-berlin.de (Vincent Froese), sepp.hartung@tu-berlin.de (Sepp Hartung), andre.nichterlein@tu-berlin.de (André Nichterlein),

rolf.niedermeier@tu-berlin.de (Rolf Niedermeier), nimrodtalmon77@gmail.com (Nimrod Talmon)

${ }^{1}$ Supported by the DFG, project PAWS (NI 369/10).

${ }^{2}$ Supported by the DFG, project DAMM (NI 369/13).

${ }^{3}$ Supported by DFG, RTG "Methods for Discrete Structures" (GRK 1408).
} 
[10] for an extended version) pioneered degree-based identity anonymization in graphs, which recently developed into a very active research field $[2,4,7,8,9$, $20,22,21,27,31]$ with theoretical as well as practical work. So far, the most frequently studied models have relied on edge modifications (allowing either only edge addition or both edge addition and deletion) [7, 10, 22, 26, 27, 31]. We are aware of one theoretical work [4] that considers vertex deletion as modification operation; there mostly computational hardness results have been achieved. We are also aware of another theoretical work [20] that considered graph contractions as modification operation. Chester et al. [8] started to investigate vertex addition, and we follow this last line of research.

There is good reason why vertex addition may be preferred to other graph modification operations when aiming at $k$-anonymity. The central point here is the "utility" of the anonymized graph. For instance, in the edge addition scenario, inserting a new edge always destroys distance properties between vertices and indeed may introduce undesirable and misleading "fake relations". Adding new vertices and connecting them to some of the vertices of the original graph could avoid this problem and gives at least a better chance to preserve essential graph properties such as connectivity, shortest paths, or diameter. For example, adding a new vertex and connecting it to only one existing vertex does not change distances between any existing vertices. Chester et al. [8] provided a more thorough discussion of the benefits of vertex addition.

The basic decision version of the vertex addition problem we study is as follows.

\section{Degree Anonymization (VA)}

Input: A simple undirected graph $G=(V, E)$ and $k, t \in \mathbb{N}$.

Question: Is there a $k$-anonymous graph $G^{\prime}=\left(V \cup V^{\prime}, E \cup E^{\prime}\right)$ such that

$$
\left|V^{\prime}\right| \leq t \text { and } E^{\prime} \subseteq\left\{\{u, v\} \subseteq V \cup V^{\prime} \mid u \in V^{\prime} \vee v \in V^{\prime}\right\} ?
$$

It is important to note that Chester et al. [8] studied a slightly different model, with decisive consequences for computational complexity: Their model gets as input a simple undirected graph $G=(V, E)$, integers $t$ and $k$, and also a vertex subset $X \subseteq V$, and the task is to $k$-anonymize the degree sequence (that is, the vertex degrees sorted in ascending order) of $X \cup V^{\prime}$ and the degree sequence of $X$. On the contrary, we consider the simpler model where $X=V$, and we require to $k$-anonymize only the degree sequence of $X \cup V^{\prime}\left(=V \cup V^{\prime}\right)$.

To better understand the difference in the models, consider the example depicted in Figure 1: In this example, the minimum solution size for the model of Chester et al. [8] is four, while the minimum solution size for our model is two. The crucial difference is that in the solution for our model, the new vertex and the old vertex of degree five together will form a 2-anonymized "block". Nevertheless, we conjecture that our results (both positive and negative) extend to the model of Chester et al. [8].

Our Contributions. Partially answering an open question of Chester et al. [8], we show that Degree Anonymization (VA) is weakly NP-hard for a compact encoding of the input. Based on this encoding, we provide several (fixed-parameter) 

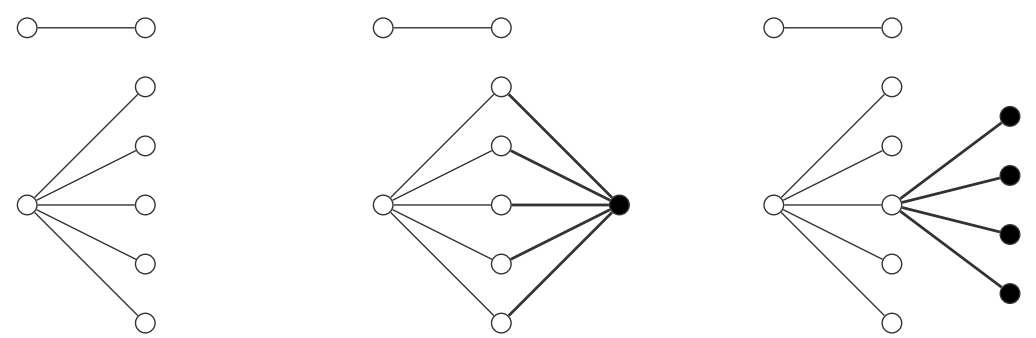

Figure 1: An example showing the difference between our model and the model of Chester et al. [8]. In this example, $k=2$, and $X=V$. The input graph on the left is not 2-anonymous. The graph in the middle is a minimum solution for our model, using only one additional vertex, while the graph on the right is a minimum solution for the model of Chester et al. [8], using four addition vertices.
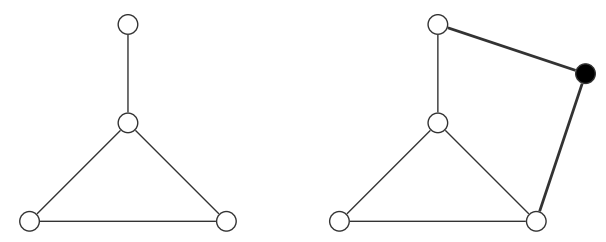

Figure 2: Degree Anonymization (VA): The input graph on the left is not 2-anonymous. The graph on the right is a possible solution for anonymizing by vertex addition. The new vertex (black) is arbitrarily connected to some other vertices.

tractability results, exploiting parameterizations by the maximum vertex degree of the input graph, the number of added vertices, and the maximum number of (implicitly) added new edges. The tractability result regarding the parameter maximum number of (implicitly) added new edges is given by developing a $b i k$ ernelization $[1,24]$ to a closely related number problem. This is one of our most technical results. Moreover, we also study variants of Degree AnonymizATION (VA) where we only allow "cloned" vertices to be added ${ }^{4}$ (that is, identical copies of existing vertices with exactly the same neighborhood; we denote this problem variant by Degree Anonymization (VC); see Figure 3 for an example) or we explicitly demand the preservation of some desirable features of the input graph (expressed by $\Pi$ ) such as distance properties (this problem variant is denoted by П-Preserving Degree Anonymization (VA)). For these practically interesting variants, we prove computational hardness already for very restricted cases (for instance even on trees). Table 1 surveys most of our results, and some open questions.

Organization of Our Work. Preliminaries and problem definitions are given in Section 2. In Section 3, we consider degree anonymization by vertex addition, where there are some specific constraints on the allowed edges connecting the

\footnotetext{
${ }^{4}$ The cloning operation is frequently studied in the context of privacy, see, for example, the work by Bilge et al. [3]. It is also studied in other contexts, for example, in social choice [12].
} 

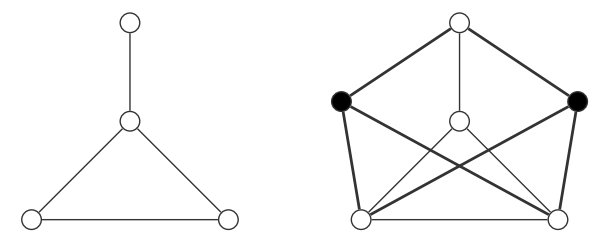

Figure 3: Degree Anonymization (VC): The input graph on the left is not 2-anonymous. The graph on the right is a possible solution for anonymizing by vertex cloning. The two added vertices (black) are clones of the middle vertex. Note that it is not possible to 2-anonymize the graph by adding only one clone.

Table 1: Overview of our results: Each column represents a different problem variant, where VC (respectively $\Pi$, VA) stands for Degree Anonymization (VC) (respectively П-Preserving Degree Anonymization (VA), Degree Anonymization (VA)). The first row refers to standard complexity analysis, while the remaining rows show parameterized complexity results with respect to several parameters. Here, $\Delta$ denotes the maximum vertex degree of the input graph, $k$ is the degree of anonymity, $s$ is the maximum number of added edges, and $t$ is the maximum number of added vertices.

\begin{tabular}{|c|c|c|c|}
\hline parameter & $\mathrm{VC}$ & $\Pi$ & VA \\
\hline- & NP-h. [Th. 1] & NP-h. [Th. 3] & weakly NP-h. [Th. 6] \\
\hline$\Delta$ & $\begin{array}{l}\text { NP-h. [Th. 1] } \\
(\Delta=3)\end{array}$ & open & open \\
\hline$k$ & $\begin{array}{l}\text { NP-h. }{ }^{a}[\text { Th. } 2] \\
(k=2)\end{array}$ & $\begin{array}{l}\text { NP-h. }{ }^{a} \text { [Th. 3] } \\
(k=2)\end{array}$ & open \\
\hline$s$ & open & W[1]-h. ${ }^{b}$ [Th. 4] & FPT [Th. 10] \\
\hline$t$ & W[2]-h. [Th. 2] & W[2]-h. [Th. 3] & $\mathrm{XP}^{c}$ [Th. 7] \\
\hline$(\Delta, k)$ & open & open & FPT [Th. 9] \\
\hline$(\Delta, t)$ & open & open & FPT [Th. 8] \\
\hline$(k, t)$ & W[2]-h. [Th. 2] & W[2]-h. [Th. 3] & $\mathrm{XP}^{c}[$ Th. 7$]$ \\
\hline
\end{tabular}

${ }^{a}$ Even on trees.

${ }^{b}$ Only for $\Pi=$ Distances.

${ }^{c}$ Open whether in FPT.

newly added vertices (including degree anonymization by vertex cloning). Here, we give strong hardness results (sometimes, even on trees). Then, in Section 4, we move on to consider degree anonymization by vertex addition, without constraints on the allowed edges connecting the newly added vertices. Here, we solve some easy cases in Section 4.1, then we give a hardness result in Section 4.2, accompanied by some tractable cases in Section 4.3. We conclude in Section 5.

\section{Preliminaries}

We consider simple undirected graphs $G=(V, E)$ where $n$ denotes the number of vertices. We denote by $\operatorname{deg}(v)$ the degree of a vertex $v \in V$ and by $\Delta:=\max _{v \in V} \operatorname{deg}(v)$ the maximum degree of $G$. For an integer $0 \leq i \leq \Delta$, we define $B_{i}:=\{v \in V \mid \operatorname{deg}(v)=i\}$, the block of degree $i$. We say that $B_{i}$ is empty 
$($ full $)$ if $B_{i}=\emptyset\left(\left|B_{i}\right| \geq k\right)$. For a full block $B_{i}$, we say that it has $z:=\left|B_{i}\right|-k$ many spare vertices. We call a block $B_{i} \operatorname{good}$ if it is empty or full, otherwise we call it bad (that is, $0<\left|B_{i}\right|<k$ ). The block sequence $B(G):=\left\{\left(i,\left|B_{i}\right|\right) \mid B_{i} \neq \emptyset\right\}$ of $G$ contains the degrees and sizes of each non-empty block. We call a block sequence realizable if it is the block sequence of a graph. For any graph $G$ and for any pair of vertices $u$ and $v$, we define $\operatorname{dist}_{G}(u, v)$ to be the length of the shortest path between $u$ and $v$ in $G$ (and $\operatorname{dist}_{G}(u, v)=\infty$ if there is no path connecting $u$ and $v$ in $G$ ). For $n \in \mathbb{N}$, we define $[n]:=\{1,2, \ldots, n\}$.

Problem Definitions. Degree Anonymization (VA) allows to add vertices and edges incident to the new vertices. For a given solution of a yes-instance, we denote the actual number of new vertices by $t^{\prime}$ and the total number of newly inserted edges by $s^{\prime}$ (indeed, $0 \leq t^{\prime} \leq t$ and $0 \leq s^{\prime} \leq s$ ).

I-Preserving Degree Anonymization (VA) defines some constraints on the new edges. The idea is to preserve some desirable properties of the input graph. A general definition reads as follows.

\section{I-Preserving Degree Anonymization (VA)}

Input: $\quad$ An undirected graph $G=(V, E)$ and $k, t \in \mathbb{N}$.

Question: Is there a $k$-anonymous graph $G^{\prime}=\left(V \cup V^{\prime}, E \cup E^{\prime}\right)$ such that $\left|V^{\prime}\right| \leq t, E^{\prime} \subseteq\left\{\{u, v\} \subseteq V \cup V^{\prime} \mid u \in V^{\prime} \vee v \in V^{\prime}\right\}$, and $\Pi$ is preserved?

We now discuss what " $\Pi$ is preserved" means for three properties we consider here. First, we say that the connectedness remains preserved if any pair of disconnected vertices in $G$ remains disconnected in $G^{\prime}$. As introducing vertices and edges cannot disconnect vertices, this property can be formalized as $\forall u, v \in V: \operatorname{dist}_{G}(u, v)=\infty \Longleftrightarrow \operatorname{dist}_{G^{\prime}}(u, v)=\infty$. Second, we say that the distances remain preserved if, for any pair of vertices in $G$, their distance is the same in $G$ and $G^{\prime}$, formally, $\forall u, v \in V: \operatorname{dist}_{G}(u, v)=\operatorname{dist}_{G^{\prime}}(u, v)$. Third, we say that the diameter remains unchanged if the diameter of $G$ and $G^{\prime}$ is the same, formally, $\max _{u, v \in V} \operatorname{dist}_{G}(u, v)=\max _{u, v \in V \cup V^{\prime}} \operatorname{dist}_{G^{\prime}}(u, v)$. Note that the diameter property also considers paths between newly added vertices, whereas this is not the case for the first two properties. The reason for this is that the diameter is naturally defined as a single number, whereas the other properties store information for each pair of vertices.

A further restricted variant of Degree Anonymization (VA) is to use vertex cloning for modifying the graph. Here, cloning a vertex $v$ means to introduce a new vertex $v^{\prime}$ and make $v^{\prime}$ adjacent to all neighbors of $v$. Formally, we arrive at the following problem:

\section{Degree Anonymization (VC)}

Input: $\quad$ An undirected graph $G=(V, E)$ and $k, t \in \mathbb{N}$.

Question: Can $G$ be transformed into a $k$-anonymous graph by at most $t$ vertex cloning operations?

We remark that there are different cloning variants: Consider two adjacent vertices $u$ and $v$. If both $u$ and $v$ are cloned, then although the clone $u^{\prime}$ is 
adjacent to $v$ and the clone $v^{\prime}$ is adjacent to $u$, the clones $u^{\prime}$ and $v^{\prime}$ may or may not be adjacent depending on the variant. If the clones are inserted simultaneously at the same time, then $u^{\prime}$ and $v^{\prime}$ are not adjacent. If the clones are inserted one after the other, then $u^{\prime}$ and $v^{\prime}$ are adjacent (no matter in which order they are inserted). Our results for Degree Anonymization (VC) (Theorems 1 and 2) hold for both variants.

Parameterized Complexity. An instance $(I, k)$ of a parameterized problem consists of the actual instance $I$ and an integer $k$ being the parameter [11, 16, 29]. A parameterized problem is called fixed-parameter tractable (FPT) if there is an algorithm solving it in $f(k) \cdot|I|^{O(1)}$ time, whereas an algorithm with running time $O\left(|I|^{f(k)}\right)$ only shows membership in the class XP (clearly, FPT $\subseteq \mathrm{XP}$ ).

A core tool in the development of fixed-parameter algorithms is polynomialtime preprocessing by data reduction, called kernelization ${ }^{5}[18,24]$. Here, the goal is to transform a given problem instance $(I, k)$ in polynomial time into an equivalent instance $\left(I^{\prime}, k^{\prime}\right)$ whose size is upper-bounded by a function of $k$. That is, $(I, k)$ is a yes-instance if and only if $\left(I^{\prime}, k^{\prime}\right), k^{\prime} \leq g(k)$, and $\left|I^{\prime}\right| \leq g(k)$ for some function $g$. Thus, such a transformation is a polynomial-time self-reduction with the constraint that the reduced instance is "small" (measured by $g(k)$ ). In case that such a transformation exists, $\left(I^{\prime}, k^{\prime}\right)$ is called kernel of size $g(k)$. Usually, a kernel is achieved by exhaustively applying a set of reduction rules, each of them transform an instance to another instance, while shrinking the instance of simplifying it. A reduction rule is said to be correct if the transformed instance is always equivalent to the original instance, that is, either both are yes-instances or both are no-instances.

One can show that a parameterized problem $L$ is (presumably) not fixedparameter tractable with a parameterized reduction from a W[1]-hard or W[2]hard problem (such as Clique or SET Cover parameterized by solution size) to $L$. A parameterized reduction from a parameterized problem $L$ to another parameterized problem $L^{\prime}$ is a function that, given an instance $(I, k)$, computes in $f(k) \cdot|I|^{O(1)}$ time an instance $\left(I^{\prime}, k^{\prime}\right)$ (with $\left.k^{\prime} \leq g(k)\right)$ such that $(I, k) \in L \Leftrightarrow$ $\left(I^{\prime}, k^{\prime}\right) \in L^{\prime}$.

\section{Constrained Degree Anonymization}

Cloning seems a natural and well-motivated modification operation for social networks. Unfortunately, we face computational intractability even on very restricted input graphs with maximum degree three. The corresponding parameterized reduction is from InDEPENDENT SET.

Theorem 1. Degree Anonymization (VC) is NP-hard, even on graphs with maximum degree three.

\footnotetext{
${ }^{5}$ It is well-known that a decidable parameterized problem is fixed-parameter tractable if and only if it has a kernelization. [6]
} 
Proof. We provide a reduction from IndePEndent SET which remains NP-hard even on cubic graphs (that is, 3-regular graphs) [17]. Given a simple undirected graph $G$ and a positive integer $h$, the INDEPENDENT SET problem asks whether $G$ contains an independent set (a set of pairwise non-adjacent vertices) of size at least $h$.

Let $G=(V, E)$ be a cubic graph. First, we construct the incidence graph $G^{\prime}=$ $\left(V^{\prime}, E^{\prime}\right)$ of $G$, where $G^{\prime}$ is a bipartite graph with the two vertex sets $V$ and $E$ and for each edge $e=\{u, v\} \in E$, we have $\{u, e\},\{v, e\} \in E^{\prime}$. Then, we add $n+2(h+3)^{2}$ triangles $\left(K_{3}\right)$ and $(h+3)^{2}$ cliques of order four $\left(K_{4}\right)$. Finally, we set $t:=h$ and $k:=n+4(h+3)^{2}+4 h$.

It remains to show that $G$ has an independent set of size at least $h$ if and only if $G^{\prime}$ can be $k$-anonymized by cloning at most $t$ vertices.

" $\Rightarrow$ ": Let $I \subseteq V$ denote an independent set of size $h$. Denote by $G^{\prime \prime}$ the graph that results from cloning all vertices of $I$ in $G^{\prime}$. We show that $G^{\prime \prime}$ is $k$ anonymous. First, observe that $G^{\prime}$ contains exactly $n+4(h+3)^{2}$ vertices of degree three and $1.5 n+3\left(n+2(h+3)^{2}\right)$ vertices of degree two. Cloning a vertex of $I$ in $G^{\prime}$ increases the degree of three degree-two vertices by one and introduces one degree-three vertex. Furthermore, as $I$ is an independent set, the degree of no vertex is increased to four. Hence, $G^{\prime \prime}$ contains $n+4(h+3)^{2}+4 t=k$ degree-three vertices and more than $3\left(n+2(h+3)^{2}\right)>k$ degree-two vertices and thus is $k$-anonymous.

" $\Leftarrow$ ": Let $G^{\prime \prime}$ be the $k$-anonymous graph obtained from cloning $t$ vertices in $G^{\prime}$. First, observe that cloning $t$ vertices in $G^{\prime}$ can increase the maximum degree by at most $t$. Thus, $\Delta_{G^{\prime \prime}} \leq 3+t$. Next, we show that $G^{\prime \prime}$ contains no vertex of degree four or more. Assume towards a contradiction that there exists a vertex $v$ of degree four or more. As cloning one vertex $u$ can introduce at most $\Delta_{G^{\prime \prime}}+1$ vertices of degree $\operatorname{deg}_{G^{\prime \prime}}(v)$ (namely $u$ and its neighbors) and since there are no degree- $\operatorname{deg}_{G^{\prime \prime}}(v)$ vertices in $G^{\prime}$, there are at most $t\left(\Delta_{G^{\prime \prime}}+1\right) \leq h(h+4)<k$ vertices of degree $\operatorname{deg}_{G^{\prime \prime}}(v)$. This is a contradiction to the assumption that $G^{\prime \prime}$ is $k$-anonymous. Hence, all vertices in $G^{\prime \prime}$ have degree at most three.

Observe that cloning a vertex $e \in E \subseteq V^{\prime}$ or any vertex in one of the $K_{4}$ 's creates degree-four vertices and, hence, no such vertex is cloned to obtain $G^{\prime \prime}$. Cloning two vertices (or one vertex twice) of a triangle creates a vertex of degree four. Hence, at most one vertex of each triangle is cloned, creating exactly two degree-three vertices. Since cloning a vertex $v \in V \subseteq V^{\prime}$ introduces at most four degree-three vertices and $G^{\prime \prime}$ has to have at least $4 t$ degree-three vertices more than $G^{\prime}$, it follows that only vertices in $V$ are cloned to obtain $G^{\prime \prime}$. Furthermore, as cloning two vertices that are adjacent in $G$ or one vertex twice introduces a degree-four vertex, it follows that the cloned vertices form an independent set of size $t=h$ in $G$.

Also from the viewpoint of fixed-parameter algorithms, we have no good news with respect to the standard parameter "solution size" $t$, even on trees. The corresponding reduction is from SET Cover.

Theorem 2. Degree Anonymization (VC) is NP-hard and W[2]-hard with respect to the maximum number $t$ of clones, even if $k=2$ and the graph is a tree. 
Proof. We provide a reduction from the W[2]-complete SET Cover problem parameterized by the solution size [11]. Here, given a collection $\mathcal{S}=\left\{S_{1}, \ldots, S_{m}\right\}$ of sets over a universe $U=\left\{x_{1}, \ldots, x_{n}\right\}$ and a positive integer $h$, the question is whether there is a set cover of size at most $h$, that is, a subset $\mathcal{S}^{\prime} \subseteq \mathcal{S}$ such that $\bigcup_{S \in \mathcal{S}^{\prime}} S=U$. Given a Set Cover instance $I=(\mathcal{S}, U, h)$, we construct a graph $G=(V, E)$ as follows. For each $x_{i} \in U$, we define $f\left(x_{i}\right):=(h+3) i+m$. First, for each element $x \in U$, we add a star with $f(x)$ leaves $\left(\right.$ a $\left.K_{1, f(x)}\right)$ whose center-vertex is denoted by $v_{x}$. Next, for each set $S \in \mathcal{S}$, we add a star $K_{1,2|S|}$ and a set gadget consisting of a tree of depth three. The root of the tree is $v_{S}$ with $2|S|$ child vertices, partitioned into $|S|$ parts with two vertices each. The two vertices of each part correspond to one element $x \in S$ and have $f(x)-2$ degree-one child vertices each and, thus, a degree of $f(x)-1$. Finally, we set $t=h$ and $k=2$.

It remains to show that $I$ has a set cover of size $h$ if and only if $G$ can be $k$-anonymized by cloning at most $t$ vertices. To this end, first observe, that the vertices $v_{x}$, for $x \in U$, are the only vertices violating 2 -anonymity. We denote the set containing all these vertices by $V_{X}$.

" $\Rightarrow$ ": Let $\mathcal{S}^{\prime} \subseteq \mathcal{S}$ be a set cover of size $h$. We show that cloning all vertices in the set $V_{C}:=\left\{v_{S} \mid S \in \mathcal{S}^{\prime}\right\}$ (containing the vertices corresponding to the sets in $\mathcal{S}^{\prime}$ ) results in a 2 -anonymous graph. Since $\mathcal{S}^{\prime}$ is a set cover, for every vertex $v_{x} \in V_{X}$, there exists a corresponding pair of vertices in a set-gadget whose degree is increased from $f(x)-1$ to $f(x)$ due to the cloning. Furthermore, no new degrees are introduced, as each neighbor $v$ of a cloned vertex corresponds to an element $x$ and $\operatorname{deg}_{G}(v)=f(x)-1$.

" $\Leftarrow$ ": Let $V_{C},\left|V_{C}\right| \leq t$, denote the set of cloned vertices and let $G^{\prime}$ be the resulting 2-anonymous graph. For each set $S \in \mathcal{S}, C_{S}$ denotes the set of all child vertices of $v_{S}$. Define $C_{\mathcal{S}}:=\bigcup_{S \in \mathcal{S}} C_{S}$. Furthermore, set $V_{\mathcal{S}}:=\left\{v_{S} \mid S \in \mathcal{S}\right\}$. Observe that, for each $x \in U$, the only vertices in $G$ whose degrees differ by at most $t$ from $v_{x}$ are vertices in $C_{\mathcal{S}}$. Furthermore, notice that, by construction, cloning a vertex from $V \backslash V_{\mathcal{S}}$ introduces at most one vertex having the same degree as a vertex from $V_{X}$. As cloning a vertex in $V_{\mathcal{S}}$ neither decreases the size of any block to one nor introduces a block of size one, and since every vertex in $C_{\mathcal{S}}$ has a neighbor in $V_{\mathcal{S}}$, we can assume that $V_{C} \subseteq V_{\mathcal{S}}$ (otherwise, we can replace vertices in $V_{C} \backslash V_{\mathcal{S}}$ with vertices in $V_{\mathcal{S}}$ ). Next, observe that, for each vertex $v_{x} \in V_{X}$, there has to be another vertex in $G^{\prime}$ having the same degree as $v_{x}$. Thus, the vertices in $V_{C} \subseteq V_{\mathcal{S}}$ correspond to a set cover of size at most $t=h$.

We can adjust the reduction from Theorem 2 to also work for П-PRESERVING Degree Anonymization (VA).

Theorem 3. For $\Pi \in\{$ Distances, Diameter, Connectivity $\}$, $\Pi-P R E S E R v i n g$ Degree Anonymization (VA) is NP-hard and also W[2]-hard with respect to the maximum number $t$ of added vertices, even for $k=2$. For $\Pi \in\{$ Distances, Connectivity\}, this is also true on trees. 
Proof. The proof is based on extending ideas from the proof of Theorem 2. We provide a reduction from the $\mathrm{W}[2]$-complete SET COVER problem parameterized by the solution size. Here, given a collection $\mathcal{S}=\left\{S_{1}, \ldots, S_{m}\right\}$ of sets over a universe $U=\left\{x_{1}, \ldots, x_{n}\right\}$ and a positive integer $h$, the question is whether there is a set cover of size at most $h$, that is, a subset $\mathcal{S}^{\prime} \subseteq \mathcal{S}$ of size at most $h$, such that $\bigcup_{S \in \mathcal{S}^{\prime}} S=U$. Given a SEt Cover instance $I=(\mathcal{S}, U, h)$, we construct a $\Pi$-Preserving Degree Anonymization (VA) instance as follows. First, we set $k=2$ and $t=h$. Next, for notational convenience, we define a helper function $f(i, z):=(t+1) n+(z-1) n(t+2)+i(t+2)$. Now we construct a graph $G=(V, E)$ as follows. For each element $x_{i}$, we define an element gadget containing, for each $z \in[t+1]$, a star $K_{1, f(i, z)}$ with center vertex $v_{i, z}$. For technical reasons, we further add an element safety gadget for each element $x_{i}$, containing two stars $K_{1, f(i, z)-1}$ for each $z \in[t+1]$. We also define, for each set $S_{j}$, a set gadget containing, for each element $x_{i} \in S_{j}$ and for each $z \in[t+1]$, a star $K_{1, f(i, z)-1}$ (its center denoted $v_{j, i, z}$ ). The general idea is that the element gadgets can be anonymized by connecting the set gadgets to some new vertices such that each new vertex corresponds to a set which contains the element corresponding to the element gadget. For the new vertices, in order to be $k$-anonymous, we add a safety gadget consisting of $k$ stars $K_{1, d}$ for each $d \in[(t+1) n]$.

The various gadgets are connected differently, depending on the property $\Pi$. The idea is to ensure that no new vertex can be connected to element gadgets corresponding to elements of different sets, by forcing such a connection to violate the property. For $\Pi \in\{$ Distances, Diameter, Connectivity $\}$ and a fixed $S_{j}$, we introduce a new vertex $v_{j}$ and connect it to $v_{j, i, z}$ for each $x_{i} \in S_{j}$ and each $z \in[t+1]$. We further add degree-one vertices connected to $v_{j}$ to fill up its degree to $f(n, t+2)$.

For $\Pi \in\{$ Distances, Diameter $\}$, we introduce another new vertex $u$, and connect $u$ to all of the $v_{j}$. Again, we fill up its degree to $f(n, t+2)$ by connecting new degree-one vertices to $u$.

For $\Pi=$ Diameter, we add two new vertices $v_{1}^{\prime}$ and $v_{m}^{\prime}$. We connect $v_{1}^{\prime}$ to $v_{1}$ and $v_{m}^{\prime}$ to $v_{m}$ by a path of length 5 . For each $j \in[m-1]$, we connect $v_{j}$ to $v_{j+1}$ by a path of length $t+8$. We connect $u$ to each $v_{j}$ by a path of length $\lceil((t+8) m+1) / 2\rceil$.

It remains to show that $I$ is a yes-instance if and only if $(G, k, t)$ is a yesinstance.

" $\Rightarrow$ ": Given a set cover $\mathcal{S}^{\prime}$, we add one new vertex $w_{j}$ for each $S_{j} \in \mathcal{S}^{\prime}$ and connect it to all of the $v_{j, i, z}$ for each $x_{i} \in S_{j}$ and each $z \in[t+1]$. We next show that this operation is permitted, as we do not violate $\Pi$. Indeed, consider any $S_{j}$ together with any pair of elements $x_{i_{1}}, x_{i_{2}} \in S_{j}$ and any pair $z_{1}, z_{2} \in[t+1]$ : For $\Pi=$ Connectivity, it holds that $v_{j, i_{1}, z_{1}}$ and $v_{j, i_{2}, z_{2}}$ were already connected; for $\Pi \in\{$ Distances, Diameter $\}$, it holds that $v_{j, i_{1}, z_{1}}$ and $v_{j, i_{2}, z_{2}}$ already had distance two, therefore the shortest path of no pair of vertices has been changed. Furthermore, the maximum distance in the new graph is still attained by the distance between $v_{1}^{\prime}$ to $v_{m}^{\prime}$, therefore the diameter did not change as well.

We now show that the graph is $k$-anonymous. First, note that the only 
vertices that need to be anonymized are the $v_{i, z}$. Since $\mathcal{S}^{\prime}$ is a set cover, for each $i$ and for each $z$, the degree of at least one $v_{j, i, z}$ is incremented by one, thus anonymizing the corresponding $v_{i, z}$. Also, the element safety gadgets ensure that we introduced no new bad blocks, and the safety gadget ensures that the new vertices are anonymized.

"६": Consider a new vertex $w$, two different sets $S_{j_{1}}, S_{j_{2}} \in \mathcal{S}$ and $z_{1}, z_{2} \in$ $[t+1]$. We show that $v_{j_{1}, i_{1}, z_{1}}$ and $v_{j_{2}, i_{2}, z_{2}}$ cannot both be connected to $w$ : For $\Pi=$ Connectivity, this is true because they are not connected; for $\Pi=$ Distances, this is true because the distance between them is four, and would change to two; for $\Pi=$ Diameter, this is true because this would decrease the diameter (specifically, the distance between $v_{1}^{\prime}$ and $v_{m}^{\prime}$ ) by more than $t$, and also creating a shortcut between the paths of length $t+8$ does not help.

Now, assume that $(G, k, t)$ is a yes-instance. As only $t$ new vertices are added, there exists an integer $z^{\prime} \in[t+1]$ such that no new vertex has the same degree as $v_{i, z^{\prime}}$ for any $x_{i} \in U$. Note that there is a gap of $t+1$ above each $v_{i, z^{\prime}}$. Hence, it must be anonymized from below. The only possible vertices to use are the $v_{j, i, z^{\prime}}$ and the centers of the stars in the element safety gadgets. We can assume, without loss of generality, that every element $x_{i}$ is contained in at least one set $S_{j}$ (as we can easily check if this is not the case, and return a negative answer). Therefore, if some vertex from an element safety gadget is used, we can always use another $v_{j, i, z^{\prime}}$ to anonymize $v_{i, z^{\prime}}$. We can then associate a unique set $S_{j}$ with every newly added vertex, and because all of the $v_{i, z^{\prime}}$ are anonymized, we get a set cover.

We strengthen parts of Theorem 3 (using a reduction from the $W[1]$-hard problem CLIQUE) by also showing that the problem remains intractable with respect to the typically larger parameter number $s$ of added edges. For simplicity, we consider $s$ as part of the input.

Theorem 4. For $\Pi=$ Distances, $\Pi$-Preserving Degree Anonymization (VA) is W[1]-hard with respect to the maximum number $s$ of new edges.

Proof. We give a reduction from the W[1]-complete CLIQUE problem parameterized by the clique size. Here, given a simple undirected graph $G=(V, E)$ and an integer $h$, the question is whether $G$ contains a clique of size at least $h$. Given a Clique instance $I:=(G, h)$, we construct a graph $G^{\prime}=\left(V^{\prime}, E^{\prime}\right)$ such that $G$ has a clique of size $h$ if and only if $G^{\prime}$ can be $k$-anonymized by adding at most $t$ new vertices and at most $s$ new edges, that is, an instance $I^{\prime}:=\left(G^{\prime}, k, t, s\right)$. For each $z \in[t+1]$, we define $f(z):=(t+1)(h-1)+z(t+3)$, and add a star $K_{1, f(z)}$ with center vertex $v_{z}$ to $G^{\prime}$. For each $z \in[t+1]$ and for each edge $e_{i} \in E=\left\{e_{1}, \ldots, e_{m}\right\}$, we add a vertex (denoted by $v_{z, i}$ ), and we say that $e_{i}$ is the corresponding edge of $v_{z, i}$.

For each pair $z_{1}, z_{2} \in[t+1]$ and for each pair of edges $e_{i_{1}}$ and $e_{i_{2}}$, we connect $v_{z_{1}, i_{1}}$ and $v_{z_{2}, i_{2}}$ by a path of length two, if the corresponding edges $e_{i_{1}}$ and $e_{i_{2}}$ share a common vertex as an endpoint. We add some new leaves to each $v_{z, i}$ such that the degree of each $v_{z, i}$ is changed to $f(z)-2$. We add some 
safety gadgets for the new vertices to safely fall into their block: Specifically, we create $k$ stars $K_{1,(t+1)(h-1)}$. Finally, we set $k=\left(\begin{array}{l}h \\ 2\end{array}\right)+1, t=h$, and $s=2(t+1)\left(\begin{array}{l}h \\ 2\end{array}\right)$.

It remains to show that $I$ is a yes-instance if and only if $(G, k, t)$ is a yesinstance.

" $\Rightarrow$ ": Given a clique of size $h$ in $G$, we add one new vertex for each clique vertex $v$ connecting it to all $v_{z, i}$ where the corresponding edge $e_{i}$ is incident to $v$ and inside the clique. We show that this operation is permitted, as we do not violate $\Pi$. Indeed, it holds that each pair $v_{z, i_{1}}, v_{z, i_{2}}$ whose corresponding edges $e_{i_{1}}, e_{i_{2}}$ share a common vertex already had distance two.

We now show that the graph is $k$-anonymous. First, note that the only vertices that need to be anonymized are the $v_{z}$ 's. The degree of each $v_{z, i}$ whose corresponding edge is inside the clique was incremented by exactly two, and since there are exactly $\left(\begin{array}{l}h \\ 2\end{array}\right)$ such edges, the $v_{z}$ 's are now anonymized. We can assume that $m \geq k+\left(\begin{array}{c}h \\ 2\end{array}\right)$ (as this case can be easily dealt with), and, therefore, no new blocks were introduced. Finally, the new vertices fall into the block anonymized by the safety gadgets.

" $\Leftarrow$ ": Consider a new vertex $w$, a pair $z_{1}, z_{2} \in[t+1]$, and a pair of edges $e_{i_{1}}, e_{i_{2}}$. The vertices $v_{z_{1}, i_{1}}$ and $v_{z_{2}, i_{2}}$ cannot both be connected to $w$, because the original distance between them was at least four and would be changed to two if both were connected to $w$.

Now, assume that $(G, k, t, s)$ is a yes-instance. As only $t$ new vertices are added, there exists a $z^{\prime} \in[t+1]$ such that no new vertex has the same degree as $v_{z^{\prime}}$. Since there is a gap of $t+1$ above $v_{z^{\prime}}$, it must be anonymized from below. The only possible vertices to use are the $v_{z^{\prime}, i}$. At least $\left(\begin{array}{l}h \\ 2\end{array}\right)$ of the $v_{z^{\prime}, i}$ must reach the degree of $v_{z^{\prime}}$ and because we can associate an original vertex to each new vertex, it follows that they correspond to a clique.

\section{Plain Degree Anonymization}

In this main section of our work, we study the unrestricted problem DEGREE AnONYMization (VA), without any restrictions on how to connect the new vertices to the input graph. This freedom might raise hope to find solutions more efficiently. Indeed, settling the computational complexity of DEGREE AnONYMizATion (VA) turns out to be tricky in that, on the one hand, we observe that several cases are fairly easy to solve, but we are not aware of any polynomial-time algorithm solving the problem in general. On the other hand, we can only prove weak NP-hardness for a number version of the problem.

In terms of fixed-parameter tractability, however, Degree AnONymizATION (VA) turns out to be more accessible. We obtain some fixed-parameter tractability results regarding, amongst others, certain (combined) parameters (for example, $s,(\Delta, k)$, and $(\Delta, t)$ ), for some of which we proved the cloning and property-preserving problem variants to be $\mathrm{W}$-hard.

\subsection{Easy Cases}

We start by analyzing the complexity of Degree Anonymization (VA) with respect to the two input values degree $k$ of anonymity and number $t$ of added 


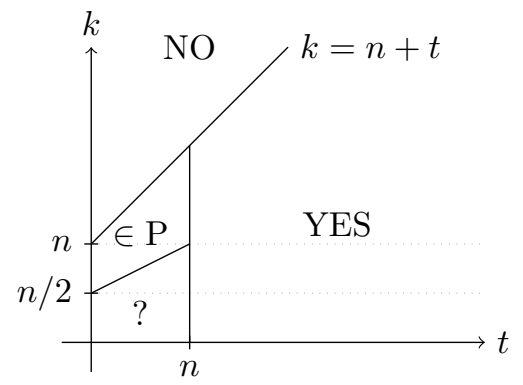

Figure 4: Visualization of our knowledge about the complexity of Degree ANONYMization (VA) depending on the degree $k$ of anonymity, the number $n$ of vertices in the input graph, and the maximum number $t$ of added vertices. The NO-cases follow from Observation 1, the YES-cases are due to Proposition 1, and the polynomial-time solvable cases follow from Proposition 2. For values inside the "?-area", the complexity is open for the graph problem (the number version is shown to be weakly NP-hard, see Theorem 6).

vertices. Figure 4 provides a two-dimensional map indicating those combinations of $k$ and $t$ for which the problem is polynomial-time solvable or even trivial. In the following, we briefly state the corresponding results, starting with the following easy observation.

Observation 1. Let $I=(G, k, t)$ be an instance of Degree AnonymizaTION (VA) with $G$ being an $n$-vertex graph. If $k>n+t$, then $I$ is a no-instance.

This holds as there are not enough vertices to make the graph anonymous, even if all vertices are in the same block, that is, the resulting graph is regular. For the other two solvable cases in Figure 4, we use the following result by Erdös and Kelly [13]:

Theorem 5 (Erdös and Kelly [13]). Let $G=(V, E)$ be a graph with $n$ vertices, maximum degree $\Delta$, and minimum degree $\delta$. Let $d \geq \Delta$ be some integer and let $\xi=\sum_{v \in V}(d-\operatorname{deg}(v))$. Then, there exists a d-regular graph $H$ with $n+t$ vertices containing $G$ as an induced subgraph if and only if

1. $t d \geq \xi$,

2. $t^{2}-(d+1) t+\xi \geq 0$,

3. $t \geq d-\delta$, and

4. $(t+n) d$ is even.

We remark that the proof given by Erdös and Kelly [13] is stated for the case $d=\Delta$ but actually proves this more general result. We also remark that the proof is constructive (indeed, it uses a result of Erdös and Gallai [14], which has a corresponding constructive version due to Hakimi [19] and Havel [23]).

First, it follows from Theorem 5 that if we are allowed to add enough new vertices (that is, at least $n$ ), then it is always possible to construct a regular graph (which is also clearly anonymous): 
Proposition 1. Let $I=(G, k, t)$ be an instance of Degree AnONymizATION (VA) with $G$ being an $n$-vertex graph. If $k \leq n+t$ and $t \geq n$, then I is a yes-instance.

Proof. We use Theorem 5 to show that for $t \geq n$ there always exists a $\Delta$ or $(\Delta+1)$-regular supergraph $H$ with $n+t$ vertices containing the input graph $G$ as induced subgraph. To this end, we check Conditions (i) to (iv) of Theorem 5. First, observe that $\xi \leq n \cdot d \leq t \cdot d$ and, thus, Condition (i) is satisfied for $d=\Delta$ and $d=\Delta+1$. Second, as $\Delta \leq n-1$ in each graph, it follows that, for $d=\Delta$ we have that

$$
t^{2}-(d+1) t+\xi \geq t(t-(n-1+1))+\xi=\xi \geq 0,
$$

and for $d=\Delta+1$ we have that

$$
t^{2}-(d+1) t+\xi \geq t(t-(n+1))+\xi \geq \xi-n \geq 0,
$$

as in the case of $d=\Delta+1$ it follows that $\xi \geq n$. Third, we have that

$$
t \geq n \geq \Delta+1 \geq d \geq d-\delta .
$$

Fourth, observe that either $(n+t) \Delta$ or $(n+t)(\Delta+1)$ is even. Hence, if we are given a Degree Anonymization (VA) instance with $t \geq n$, then it is always possible to create a regular graph with $n+t$ vertices. Thus, if $t \geq n$, then the Degree Anonymization (VA) is a yes-instance if and only if $k \leq t+n$.

We can also use Theorem 5 algorithmically, as follows.

Proposition 2. Degree Anonymization (VA) is polynomial-time solvable for $2 k>(n+t)$.

Proof. Let $I=(G=(V, E), k, t)$ be an instance of Degree AnonymizaTION (VA) with $2 k>(n+t)$. By Observation 1 and Proposition 1, we can assume that $k \leq n+t<2 k$ and $t<n$. Observe that in this case any solution (if it exists) transforms $G$ into a regular graph. Hence, the question is whether there is a regular graph $H$ with at most $n+t$ vertices containing $G$ as induced subgraph.

Our algorithm is as follows. First, we guess in $O\left(n^{2}\right)$ time the number $t^{\prime} \leq t$ of vertices that we will add and the degree $d$ of the final regular graph $H$. Then, we reduce to Theorem 5 , that is, we compute $\xi=\sum_{v \in V} d-\operatorname{deg}(v)$ and check the four inequalities.

\section{2. (Weak) NP-Hardness}

In Figure 4, we left open the computational complexity of the DegreE AnONYMization (VA) problem for instances with $2 k \leq n+t$. We now partially settle this question by proving that a closely related number version of the problem is weakly NP-hard. To this end, note that since we are not allowed to add any edges between old vertices, the actual structure of the input graph $G$ becomes negligible and we only need to store the information of how many vertices of which degree it contains (that is, its block sequence $B(G)$ ): 
Observation 2. Let $G$ and $G^{\prime}$ be two graphs with identical block sequences, that is, $B(G)=B\left(G^{\prime}\right)$. Then, for the Degree Anonymization (VA) instances $I:=(G, k, t)$ and $I^{\prime}:=\left(G^{\prime}, k, t\right)$, it holds that $I$ is a yes-instance if and only if $I^{\prime}$ is a yes-instance.

Based on Observation 2, we can now define a closely related number version of Degree Anonymization (VA).

\section{Block Sequence Anonymization (VA)}

Input: $\quad$ A realizable block sequence $B$ and $k, t \in \mathbb{N}$.

Question: Is there an undirected graph $G$ with block sequence $B$ such that $(G, k, t)$ is a yes-instance of Degree Anonymization (VA)?

Note that Block Sequence Anonymization (VA) is a pure number problem (that is, its input consists only of numbers). This helps us to develop a polynomial-time reduction from a weakly NP-hard version of the SUBSET Sum problem. An NP-hard problem is weakly NP-hard if it can be solved in polynomial-time provided that the input is encoded in unary. We obtain the following theorem.

Theorem 6. Block Sequence Anonymization (VA) is weakly NP-hard.

Proof. We reduce from the weakly NP-hard ChAnge MAKING problem [28]: Given integers $a_{1}, \ldots, a_{n}, m$, and $b$, are there nonnegative integers $x_{1}, \ldots, x_{n}$ such that $\Sigma_{i \in[n]} x_{i} \leq m$ and $\Sigma_{i \in[n]} x_{i} a_{i}=b$ ? We can assume, without loss of generality, that $\forall i \neq j:\left|a_{i}-a_{j}\right| \geq m^{3}$. If this property does not hold, then we simply multiply all numbers by $m^{3}$, that is, we set $a_{i}$ to be $m^{3} \cdot a_{i}$ and set $b$ to be $m^{3} \cdot b$. It is easy to verify that this new instance is a yes-instance if and only if the original instance is a yes-instance.

We now create an equivalent Block Sequence Anonymization (VA) instance $(B, k, t)$, with $t:=m$ and $k:=t(b+n+5 t+1)$. The realizable block sequence $B$ is the block sequence of a graph $G$, which is defined as follows. We introduce several gadgets, that is, subgraphs of $G$ with distinguished vertices of specific degrees which play an important role in the correctness proof. In the following, we only specify the degrees of these proper vertices. To build these gadgets, we add an appropriate number of degree-one neighbors. Our construction ensures that, when $k$-anonymizing $G$ by adding $t$ vertices, the degree-one vertices will always keep their degree. The construction works as follows.

Add a $b$-gadget consisting of $5 t$ base vertices of degree $n+t$, add $b$ count vertices of degree $n+2 t-1$, and add $k-b-5 t$-catch vertices of degree $n+2 t$. For each $i \in[n]$, add one $a_{i}$-gadget consisting of one $a_{i}$-vertex of degree $a_{i}+n+4 t+1$ and $k-1 a_{i}$-catch vertices of degree $a_{i}+n+5 t+1$. Finally, add a dummy gadget consisting of one dummy vertex of degree $n+4 t+1$ and $k-1$ dummy catch vertices of degree $n+5 t+1$. This completes the construction. See Figure 5 for an illustration.

We show that $\left(a_{1}, \ldots, a_{n}, m, b\right)$ is a yes-instance of ChAnge MAKING if and only if $(B, k, t)$ is a yes-instance of Block Sequence Anonymization (VA). 


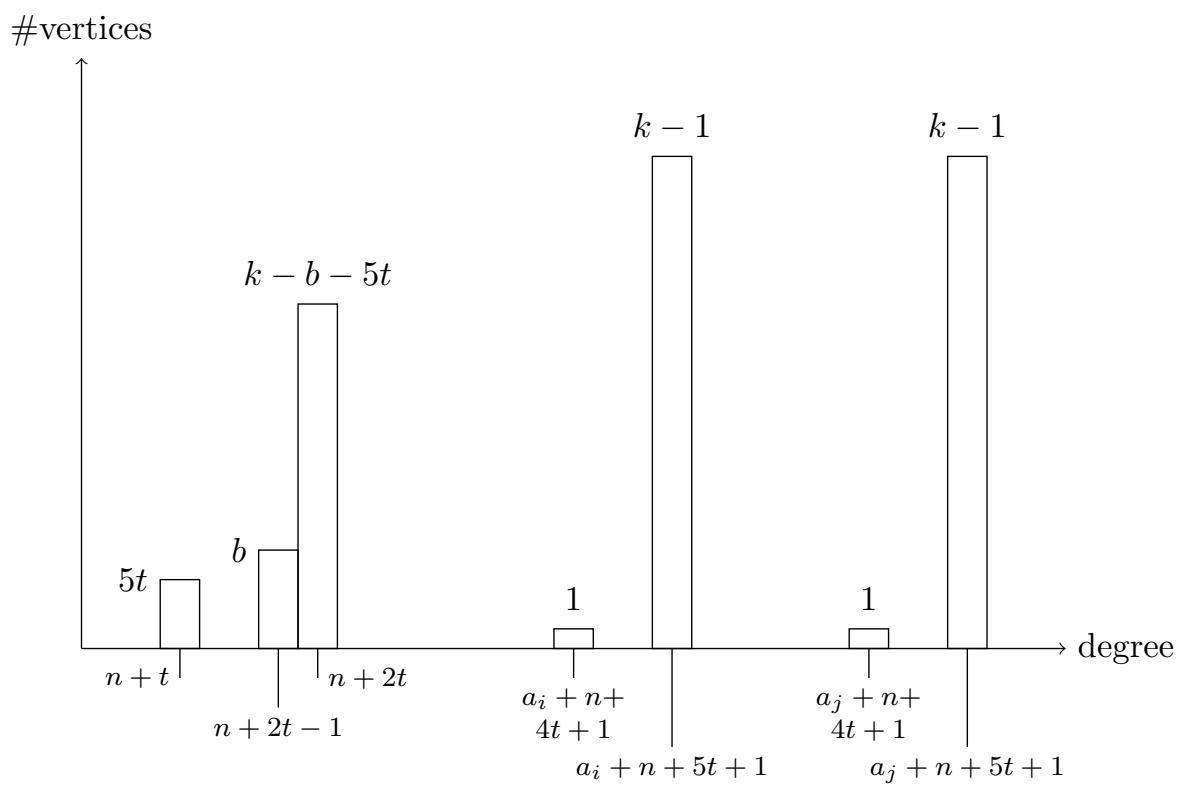

\section{\#vertices}

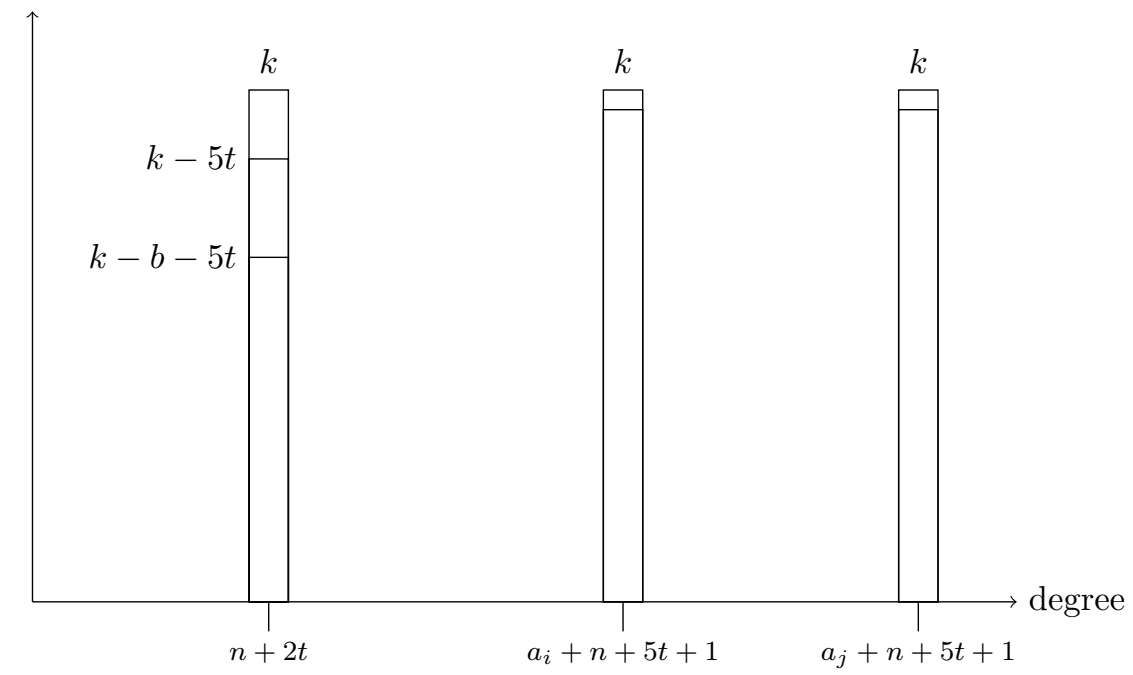

Figure 5: The reduction used in Theorem 6. The construction (corresponding to a CHANGE MAKING input) is depicted top, while an anonymized solution (corresponding to a CHANGE MAKING solution) is depicted bottom. The plots show the number of vertices in each block (that is, of each degree). 
" $\Rightarrow$ ": Assume that there are integers $x_{1}, \ldots, x_{n}$ such that $\Sigma_{i \in[n]} x_{i} \leq m$, and $\Sigma_{i \in[n]} x_{i} a_{i}=b$. We construct a $k$-anonymous graph $G^{\prime}$ by adding $t$ new vertices to $G$ as follows. For each $i \in[n]$, add $x_{i}$ new solution vertices and connect them with $a_{i}$ count vertices such that the degree of each count vertex is increased by one. (Note that this is possible because $\Sigma_{i \in[n]} x_{i} a_{i}=b$.) If $d:=t-\Sigma_{i \in[n]} x_{i}>0$, then add $d$ further new auxiliary vertices. Connect each of the new vertices (solution and auxiliary vertices) to each $a_{i}$-vertex, $i \in[n]$, to the base vertices, and to the dummy vertex.

We now claim that $G^{\prime}$ is $k$-anonymous: First, observe that all new vertices are $k$-anonymous: The auxiliary vertices are of degree $n+5 t+1$ together with $k$ further vertices from the dummy gadget. Each solution vertex is of some degree $a_{i}+n+5 t+1, i \in[n]$, together with $k-1 a_{i}$-catch vertices and one $a_{i}$-vertex. Second, the original vertices are also $k$-anonymous: All $k$ vertices from the dummy gadget get degree $n+5 t+1$ because we connected the dummy vertex to all $t$ new vertices and the degree of each dummy catch vertex remains unchanged. All $k$ vertices from the $b$-gadget get degree $n+2 t$ since we connected each base vertex to all $t$ new vertices, each count vertex with exactly one new vertex, and the degrees of the $b$-catch vertices remain unchanged. For each $i \in[n]$, all $k$ vertices of the $a_{i}$-gadget get degree $a_{i}+n+5 t+1$ since the degree of each $a_{i}$-catch vertex remains $a_{i}+n+5 t+1$ and each $a_{i}$-vertex gets degree $a_{i}+n+5 t+1$ because we connected it to all $t$ new vertices. Thus, $G^{\prime}$ is $k$-anonymous.

" $\Leftarrow$ ": Assume that there is a vertex set $V^{\prime}:=\left\{v_{1}, \ldots, v_{t^{\prime}}\right\}, t^{\prime} \leq t$, and a set of edges $E^{\prime}$ such that the graph $G^{\prime}:=\left(V \cup V^{\prime}, E \cup E^{\prime}\right)$ is $k$-anonymous. We show that $y_{1}, \ldots, y_{n}$ with $y_{i}=\left|\left\{v \in V^{\prime} \mid \operatorname{deg}_{G^{\prime}}(v)=a_{i}+n+5 t+1\right\}\right|$ is a solution for $\left(a_{1}, \ldots, a_{n}, m, b\right)$. First, we show that, for each proper original vertex from $V$, the degree in $G^{\prime}$ is already determined by the construction of $G$. To this end, recall that we can only increase the degree of each original vertex by at most $t$. We say two original vertices are close enough if their degrees in $G$ differ by at most $t$. Consider some proper vertex from the $b$-gadget, from an $a_{i}$-gadget, or from the dummy gadget, and observe that only proper vertices from the same gadget are close enough. Moreover, since, even together with $t$ potential new vertices from $V^{\prime}$, each gadget contains less than $2 k$ proper vertices, it follows that all vertices from the same gadget must end up with the same degree in $G^{\prime}$. More precisely, all vertices from the $b$-gadget must have degree $n+2 t$ in $G^{\prime}$ : Adding $V^{\prime}$ must increase the degree of each base vertex by $t$ to $n+2 t$, which is already the original degree of the $b$-catch vertices. All vertices from the dummy gadget must have degree $n+5 t+1$ in $G^{\prime}$ : Adding $V^{\prime}$ must increase the degree of the dummy vertex by $t$ to $n+5 t+1$, which is already the original degree of the dummy catch vertices. For each $i \in[n]$, all vertices from the $a_{i}$-gadget must have degree $a_{i}+n+5 t+1$ in $G^{\prime}$ : Adding $V^{\prime}$ must increase the degree of each $a_{i}$-vertex by $t$ to $a_{i}+n+5 t+1$, which is already the original degree of the $a_{i}$-catch vertices.

Second, observe that each new vertex from $V^{\prime}$ must have degree $a_{i}+n+5 t+1$ for some $i \in[n]$ or degree $n+5 t+1$ : Clearly, as we have already seen, adding $V^{\prime}$ increases the degree of all $5 t$ base vertices, of all $n a_{i}$-vertices, and of the dummy 
vertex by $t$. Thus, each new vertex has degree at least $n+5 t+1$. Since we have at most $t<k$ new vertices, each new vertex must have the same degree in $G^{\prime}$ as some proper original vertex.

Third, observe that each (non-proper) degree-one vertex from $V$ will keep degree one in $G^{\prime}$ : Only other degree-one vertices are close enough and the degree-one vertices also cannot reach the degree of any new vertex. Thus, if some original degree-one vertex has degree at least two in $G^{\prime}$, then at least $k$ original degree-one vertices must have degree at least two in $G^{\prime}$. Then, since $k=t(b+n+5 t+1)$, at least one new vertex would have degree at least $b+n+5 t+1$ in $G^{\prime}$. This is not possible because no proper original vertex can reach this degree.

Finally, recall that $y_{i}, i \in[n]$, denotes the number of new vertices from $V^{\prime}$ that have degree $a_{i}+n+5 t+1$ in $G^{\prime}$. Since adding $V^{\prime}$ must increase the degree of each of the $b$ count vertices by exactly one, there are exactly $b$ edges between new vertices and count vertices. Furthermore, let $\ell$ denote the number of edges between new vertices. It holds that $b+\ell / 2=\Sigma_{i \in[n]} y_{i} a_{i}$. More precisely,

$$
\frac{\ell}{2 m^{3}}=\frac{\Sigma_{i \in[n]} y_{i} a_{i}}{m^{3}}-\frac{b}{m^{3}}
$$

must be an integer since each $a_{i}, i \in[n]$, and $b$ are divisible by $m^{3}$. Since $0 \leq \ell<\left(\begin{array}{l}t \\ 2\end{array}\right)<m^{2}$, this is only possible if $\ell=0$. Thus, there are no edges between new vertices in $E^{\prime}$ but there exist some $y_{1}, \ldots, y_{n}$ such that $\Sigma_{i \in[n]} y_{i} a_{i}=b$. Hence, $y_{1}, \ldots, y_{n}$ is indeed a solution for $\left(a_{1}, \ldots, a_{n}, m, b\right)$.

\subsection{Tractability Results}

While it remains open whether Degree AnONymization (VA) is NP-hard, the weak NP-hardness result for Block SEquence Anonymization (VA) (Theorem 6) lets us conjecture that also the graph problem may be hard to solve. Hence, a parameterized approach solving Degree Anonymization (VA) is reasonable. Notably, we provide several (fixed-parameter) tractability results contrasting the hardness results for the constrained problem versions considered in Section 3.

A natural parameter to consider is the solution size $t$. Unfortunately, we do not know whether Degree Anonymization (VA) is fixed-parameter tractable with respect to $t$; we can only show that Degree AnONymization (VA) is polynomial-time solvable when $t$ is a constant.

Theorem 7. Degree Anonymization (VA) parameterized by the maximum number $t$ of added vertices is in XP.

Proof. Let us denote the number of edges that a new vertex $t_{i}$ has to old vertices by $d_{\text {old }}\left(t_{i}\right)$. We first guess $t^{\prime}$ (in $O(t)$ time), and $d_{\text {old }}\left(t_{i}\right)$, for each new vertex $t_{i}$ (in $O\left(n^{t}\right)$ time). We also guess the subgraph induced by the new vertices (in $O\left(2^{t^{2}}\right)$ time). Then, we use a modified version of a dynamic program used by Liu and Terzi [26]. 
The first modification is needed because we have to satisfy the guessed degrees of the new vertices when we $k$-anonymize the old vertices. Specifically, we $k$-anonymize the combined degree sequence containing the original degrees of the old vertices, and also the guessed degrees of the new vertices, with the exception that the new vertices' degrees are fixed (that is, cannot be changed, as they are already guessed). The second modification is needed to make sure that we can realize the overall increases of degrees by using exactly the guessed degrees of the new vertices. Specifically, we maintain a vector of size $t^{\prime}$ with entries upper-bounded by the guessed $d_{\text {old }}\left(t_{i}\right)$, where each entry in the vector contains the number of edges already used for the corresponding new vertex (the vector is initialized to contain only zeros). These modifications give a multiplicative factor of $O\left(n^{t}\right)$ to the running time of the dynamic programming, resulting in an overall time complexity of $O\left(f(t) \cdot k \cdot n^{t}\right)$.

We can "improve" containment in XP (as shown in Theorem 7) to fixedparameter tractability with respect to the combined parameter $(t, \Delta)$. Before proving the corresponding theorem, we introduce some notation and a helpful lemma.

For a set $A$ of vertices whose addition (together with the addition of edges) transforms a graph $G=(V, E)$ into a $k$-anonymous graph, we call $A$ an addition set and we write $G+A$ for the $k$-anonymous graph. Furthermore, the edges in $G+A$ having at least one endpoint in $A$ (the "added" edges) are denoted by $E(A)$. Hence, $G+A=(V \cup A, E \cup E(A))$.

Clearly, for an addition set $A$ of size $t$, all vertices in $G+A$, except those in $A$, have degree at most $\Delta+t$ where $\Delta$ is the largest degree in $G$. It may happen that the degree of some (potentially all) vertices from $A$ in $G+A$ is larger than $\Delta+t$. In this case, there are full blocks in $G+A$ of degree larger than $\Delta+t$ consisting only of vertices from $A$, implying that $t \geq k$. We call blocks corresponding to degrees greater than $\Delta+t$ large-degree blocks. Lemma 1 shows that we may assume that there are at most two large-degree blocks which are, in terms of their degree values, not "too far away" from each other.

Lemma 1. Let $(G, k, t)$ be a yes-instance of Degree Anonymization (VA). There is an addition set $A$ of size at most $t$ such that in $G+A$ there are at most two large-degree blocks. Furthermore, if there are two large-degree blocks, then their degrees differ by exactly one.

Proof. Let $(G, k, t)$ be a yes-instance of Degree Anonymization (VA) and let $A$ be a corresponding addition set. Assume that there are at least two large-degree blocks $B_{i}$ and $B_{j}$ in $G+A$ with $|j-i|>1$. We restructure $E(A)$ to obtain a solution as desired. To this end, we introduce some notation. Let $A^{L}=\left\{a_{1}, \ldots, a_{\ell}\right\}$ denote the set of vertices in the large-degree blocks. Observe that $\ell \geq 2 k$. For any vertex $v \in(V \cup A) \backslash A^{L}$, denote by $\operatorname{deg}^{L}(v)$ the number of neighbors of $v$ in $A^{L}$, formally $\operatorname{deg}^{L}(v):=\left|N_{G+A}(v) \cap A^{L}\right|$.

The idea is to restructure the graph such that each old vertex will be adjacent to the same number of new vertices, but the new vertex degrees will be closer to each other. We achieve this by a round-robin method. Specifically, the 
restructuring is done as follows: First, remove all edges that have at least one endpoint in $A^{L}$. Let $v_{1}, \ldots, v_{n+|A|-\ell}$ be an arbitrary ordering of vertices in $(V \cup A) \backslash A^{L}$. Then, we add edges in several steps such that in step $i$, vertex $v_{i} \in(V \cup A) \backslash A^{L}$ gets $\operatorname{deg}^{L}\left(v_{i}\right)$ incident edges, that is, overall the degree of $v_{i}$ will remain unchanged. For every $a \in A^{L}$, denote by $\operatorname{deg}_{i}^{L}(a)$ the degree of $a$ before step $i$. Then, let $X_{i}$ denote the $\operatorname{deg}^{L}\left(v_{i}\right)$ vertices in $A^{L}$ having the smallest value $\operatorname{deg}_{i}^{L}(a)$. In step $i$, we make $v_{i}$ adjacent to all vertices in $X_{i}$. Observe that, since $\operatorname{deg}^{L}\left(v_{i}\right) \leq \ell$, in each step it holds for all $j, j^{\prime} \in\{1, \ldots, \ell\}$ that $\left|\operatorname{deg}_{i}^{L}\left(a_{j}\right)-\operatorname{deg}_{i}^{L}\left(a_{j^{\prime}}\right)\right| \leq 1$. Hence, after the last step, there are at most two large-degree blocks $B_{d}$ and $B_{d+1}$ whose degrees differ by at most one.

It remains to ensure that $\left|B_{d}\right| \geq k$ and $\left|B_{d+1}\right| \geq k$. If $\left|B_{d}\right|$ is even, then add a perfect matching on the vertices in $B_{d}$ resulting in one large block of size $\ell \geq 2 k$. Otherwise, if $\left|B_{d}\right|<\left|B_{d+1}\right|$, then set $d^{\prime}:=d+1$ and add edges to introduce a Hamilton cycle in $G\left[B_{d}\right]$, increasing the degree of every vertex in $B_{d}$ by two. If $\left|B_{d}\right| \geq\left|B_{d+1}\right|$, then set $d^{\prime}:=d$. Now, add a matching between vertices in $B_{d^{\prime}}$ such that after adding these edges it holds that $0 \leq$ $\left|B_{d^{\prime}}\right|-\left|B_{d^{\prime}+1}\right| \leq 2$. Observe that this is always possible as $\left|B_{d^{\prime}}\right| \geq\left|B_{d^{\prime}+1}\right|$. We claim that $\left|B_{d^{\prime}}\right| \geq k$ and $\left|B_{d^{\prime}+1}\right| \geq k$. Suppose towards a contradiction that $\left|B_{d^{\prime}+1}\right|<k$. Since $\left|B_{d^{\prime}+1}\right| \leq\left|B_{d^{\prime}}\right| \leq\left|B_{d^{\prime}+1}\right|+2$ and $\left|B_{d^{\prime}+1}\right|+\left|B_{d^{\prime}}\right|=\ell \geq 2 k$, it follows that $\ell=2 k,\left|B_{d^{\prime}+1}\right|=k-1$, and $\left|B_{d^{\prime}}\right|=k+1$. Furthermore, as we already handled the case where $\left|B_{d}\right|$ was even, it follows that $\left|B_{d^{\prime}}\right|$ and $\left|B_{d^{\prime}+1}\right|$ are odd and $k$ is even. Also, observe that we started with the assumption that there is a solution with at least two large blocks $B_{i}$ and $B_{j}$. Since $\ell=2 k$, there are exactly two large blocks in this solution. Thus, the sum of the degrees of all vertices in $A^{L}$ is $\left|B_{i}\right| \cdot i+\left|B_{j}\right| \cdot j$ which is an even number as $\left|B_{i}\right|=\left|B_{j}\right|=k$ is even. However, as $\left|B_{d^{\prime}}\right|$ and $\left|B_{d^{\prime}+1}\right|$ are odd, the sum of all degrees of vertices in $A^{L}$ after the restructuring is $\left|B_{d^{\prime}}\right| \cdot d^{\prime}+\left|B_{d^{\prime}+1}\right| \cdot\left(d^{\prime}+1\right)$ which is odd as either $d^{\prime}$ or $d^{\prime}+1$ is odd. This is a contradiction to the fact that our restructuring did not change the degrees of vertices in $(V \cup A) \backslash A^{L}$ and our operations (edge deletions and insertions) do not change the parity of the sum of the degrees of the vertices in $A^{L}$.

Using Lemma 1, we can now prove fixed-parameter tractability for the parameter combination $(t, \Delta)$; however, the result uses Lenstra's result about the fixed-parameter tractability of integer linear programs [25], thus making it of mainly theoretical interest only.

Theorem 8. Degree Anonymization (VA) is fixed-parameter tractable with respect to the combined parameter $(t, \Delta)$.

Proof. Our algorithm consists of three phases. First (Phase I), we guess what the solution looks like, specifically guessing the degrees of the good blocks, and the degrees of the new vertices, while respecting the guessed degrees of the good blocks. Then (Phase II), we use a bottom-up "lazy" method to solve the instance for the old vertices, respecting the guessed degrees of the new vertices. Finally (Phase III), we use integer linear programming to solve the instance for the new vertices. A detailed description follows. 
Phase I: We guess the subgraph induced by the new vertices (in $O\left(2^{t^{2}}\right.$ ) time). We know, from Lemma 1, that the number of possible blocks in the solution is upper-bounded by $\Delta+t+2 \in O(\Delta+t)$. We guess the degrees of the two large-degree blocks (in $O(n+t)$ time). Then, we guess, for each block, whether it is empty or full (in $O\left(2^{\Delta+t}\right)$ ). Finally, we guess the degree of each new vertex (in $O(\Delta+t)^{t}$ time). Phase I runs in $O\left((n+t) \cdot 2^{t^{2}} \cdot 2^{\Delta+t} \cdot(\Delta+t)^{t}\right)=$ : $O\left((n+t) \cdot f_{1}(t, \Delta)\right)$ time.

For ease of presentation, we say that we move a vertex up, meaning that we connect it to some new vertices, thus changing its degree and moving it to a different block of some desired degree. We can choose which new vertices to use in a round-robin way, subject to their guessed degrees (that is, each new vertex participates in the round-robin until it reaches its guessed degree).

Phase II: We perform the following bottom-up lazy method. We start from the lowest degree block, and work all the way up to the highest degree block. If the current block $B_{i}$ is guessed to be empty, then we move its vertices up, to the first block above it which is guessed to become full (if there is a gap greater than $t$ to such a block, then we continue with the next iteration). Otherwise, if it is guessed to be full, then we distinguish between the following two cases: If the number of old vertices in the block plus the number of new vertices guessed to be in this block is at least $k$, then we do nothing because it means that this block is already anonymized, and continue with the next block. Otherwise, $B_{i}$ has a shortage of some $z_{i}$ many vertices to become full, so we find the maximum $j<i$ such that the number of old vertices in $B_{j}$ plus the number of new vertices guessed to be in $B_{j}$ is greater than $k$ (specifically, equals $k+z_{j}$ for some $z_{j}$ spare vertices in $B_{j}$; if the gap $i-j$ is greater than $t$, then we continue with the next iteration because $B_{i}$ cannot be $k$-anonymized). We move $\min \left(z_{i}, z_{j}\right)$ spare vertices from $B_{j}$ to $B_{i}$. If, after moving these spare vertices, $B_{i}$ still needs some more vertices (that is, if $z_{i}>z_{j}$ ), then we repeat this step once more, looking for the maximum $j^{\prime}<j$ such that the number of old vertices in $B_{j^{\prime}}$ plus the number of new vertices guessed to be in $B_{j^{\prime}}$ is greater than $k$, until we have enough vertices in the current block. If in the end of this phase all of the blocks are anonymized, then we continue with the next phase. The overall time cost of Phase II is in $O(\Delta+t)^{3}=: O\left(f_{2}(t, \Delta)\right)$.

Our approach is lazy because we are performing the minimum amount of changes to make the old vertices anonymous. First, we use the spare vertices from the closest full block below the current one. Second, we move the minimum number of vertices to make the blocks anonymized with respect to the old vertices, that is, we only make the bad blocks full, but never overfull.

Phase III: We check whether each new vertex reached its guessed degree. If so, then we halt with a positive answer. If there are new vertices which do not yet have their guessed degrees, then we still have some hope of reaching these degrees, because of the laziness of Phase II, so we try to move up some more old vertices, until we reach the guessed degrees, while not destroying the anonymity of the blocks. To this end, denote the number $\left|B_{i}\right|-k$ of spare vertices in each full block $B_{i}$ by $z_{i}$. Note that we can move any number of up to $z_{i}$ vertices from 
this block, to any full block above it, and no other moves are possible. Now, our problem reduces to the following integer linear program:

Input: Integers $\left\{z_{1}^{\prime}, \ldots, z_{n^{\prime}}^{\prime}\right\}$, an $n^{\prime} \times m^{\prime}$ matrix $A=a_{i j}$, and an integer $Z$.

Task: Maximize $\sum_{i \in\left[n^{\prime}\right]} \sum_{j \in\left[m^{\prime}\right]} a_{i j} x_{i j}$ such that $\sum_{i \in\left[n^{\prime}\right]} \sum_{j \in\left[m^{\prime}\right]} a_{i j} x_{i j} \leq Z$ and $\forall j: \sum_{i \in\left[n^{\prime}\right]} a_{i j} \leq z_{j}$.

Specifically, we set $n^{\prime}$ and $m^{\prime}$ to be the number of full blocks (that is, $n^{\prime}=$ $\left.m^{\prime} \in O(\Delta+t)\right)$. For each full block, we set $z_{i}^{\prime}$ to be $z_{i}$ and $a_{i j}$ to be the gap between the $j^{\text {th }}$ full block and the $i^{\text {th }}$ full block. We set $Z$ equal to the overall sum of differences of guessed degrees of the new vertices and their degrees after Phase II. Note that any solution to the integer linear program is realizable as each $a_{i j}$ is upper-bounded by the number of spare vertices $z_{j}$. Moreover, the number of variables is upper-bounded by the number of full blocks squared, that is, by $O\left((\Delta+t)^{2}\right)$. By a famous result of Lenstra [25], it follows that the running time is exponential only in the number of variables, therefore the cost of this phase is in $\operatorname{poly}(n, t) \cdot f_{3}(t, \Delta)$, for some computable function $f_{3}$.

We now prove the correctness of the algorithm. As the algorithm only performs permitted operations (that is, adds up to $t$ new vertices and connects them such that each edge is incident to at least one new vertex), it follows that if the input is a no-instance, then the algorithm returns a negative answer. Otherwise, if the input is a yes-instance, then at least one set of guesses from Phase I will be correct. Any solution must at least move the vertices that are moved in Phase II, and then the problem reduces to the integer linear program presented in Phase III.

The question whether fixed-parameter tractability also holds for the single parameters $t$ or $\Delta$ remains open. Nevertheless, we find that fixed-parameter tractability also holds for the combined parameter $(\Delta, k)$.

Theorem 9. Degree AnONymization (VA) is fixed-parameter tractable with respect to the combined parameter $(\Delta, k)$.

Proof. It follows, from Theorem 8 that if $t \leq(\Delta k+k) \cdot(\Delta+1)^{2}$, then we are done; therefore, we assume that $t>(\Delta k+k) \cdot(\Delta+1)^{2}$. If $\Delta=0$, then the input graph is already anonymized; therefore, we assume that $\Delta>0$. If $k \geq n$, then we can solve the input instance in polynomial time by Observation 1, Proposition 1 , and Proposition 2. Hence, we assume $k<n$.

Consider the following method for $k$-anonymizing the graph, showing that we can answer positively for any remaining instance. For each block $B_{i}$, if $\left|B_{i}\right|<\Delta k+k$, then we connect each vertex in $B_{i}$ to $\Delta+1-i$ new vertices such that the degree of each old vertex in $B_{i}$ becomes $\Delta+1$ and the degree of each new vertex becomes one. If $\left|B_{i}\right| \geq \Delta k+k$, we do the same, but only for $\Delta k$ arbitrarily chosen vertices in $B_{i}$.

The resulting graph has exactly two non-empty blocks: $B_{1}$ containing all of the new vertices (and only them) and $B_{\Delta+1}$ filled with all of the old vertices (and only them). Since $k<n=\left|B_{\Delta+1}\right|$, the block $B_{\Delta+1}$ is good. 
Let $t^{\prime}:=\left|B_{1}\right|$ denote the number of new vertices added by our method. There are at most $\Delta+1$ blocks in the original graph and we move at most $\Delta k+k$ vertices from each block by at most $\Delta+1$. Therefore, $t^{\prime} \leq(\Delta k+k) \cdot(\Delta+1)^{2} \leq t$. If there are no blocks of size greater than $\Delta k+k$ in the original graph, then $t^{\prime} \geq n>k$ because we moved all of the old vertices. Otherwise, if there is at least one block of size greater than $\Delta k+k$, then $t^{\prime} \geq \Delta k \geq k$ because we moved at least $\Delta k$ vertices from this block by at least one block.

Contrasting the W[1]-hardness of П-Preserving Degree AnONymizaTION (VA) parameterized by the maximum number $s$ of new edges (Theorem 4), we conclude with fixed-parameter tractability for DEGREE ANONYMIZATION (VA) with respect to $s$. We again assume that $s$ is given as part of the input.

Theorem 10. Degree Anonymization (VA) is fixed-parameter tractable with respect to the maximum number $s$ of newly inserted edges.

To prove Theorem 10, we define the problem of anonymizing a general (not necessarily realizable) block sequence by vertex addition. Indeed, the fixed-parameter tractability of Degree Anonymization (VA) directly follows by providing a so-called bikernelization to this new problem (informally, a bikernelization is a kernelization to a different problem).

A general block sequence is a tuple $\mathcal{B}=\left(b_{0}, \ldots, b_{d}\right)$ of nonnegative integers $b_{i} \geq 0$. We say that $b_{i}$ denotes the size of the block of degree $i \in\{0, \ldots, d\}$ and we denote the length of $\mathcal{B}$ by $|\mathcal{B}|$. We consider tuples of integers $x=$ $\left(x_{0}, \ldots, x_{d}\right)$, where $0 \leq x_{i} \leq b_{i}$ for all $i \in\{0, \ldots, d\}$, and as we usually think about these tuples as corresponding to the new vertices that we add in the anonymization process, we call such a tuple a vertex. We denote the requirement that $0 \leq x_{i} \leq b_{i}$ for all $i \in\{0, \ldots, d\}$, by writing $x \leq \mathcal{B}$. We define now what it means to add the vertex $x$ to the general block sequence $\mathcal{B}$. Intuitively, for each $x=\left(x_{0}, \ldots, x_{d}\right), x_{i}$ denotes the number of degree- $i$ vertices to which the "newly added vertex" $x$ is connected. We denote the resulting general block sequence by $\mathcal{B} \oplus x$. Before giving the definition, we make a technical disclaimer: whenever we use an index $i$ that is not contained in $\mathcal{B}$ (that is, $i>d$ ), then we implicitly assume that $\mathcal{B}$ is extended to length $i$ by appending the corresponding number of zero entries to $\mathcal{B}$.

The general block sequence $\mathcal{B} \oplus x$ is generated out of $\mathcal{B}$ by iteratively performing the following operations, for each $i \in\{0, \ldots, d\}$ : decrease $b_{i}$ by $x_{i}$ and increase $b_{i+1}$ by $x_{i}$. These replacements correspond to increasing the degrees of the specified number of original vertices that are connected to the new vertex by one. Moreover, in order to insert the new vertex, let $\delta(x):=\sum_{i=0}^{|x|} x_{i}$ be its degree and increase $b_{\delta(x)}$ by one. Note that the number of added edges equals $\delta(x)$. For a sequence of vertices $\left(x^{1}, \ldots, x^{t}\right)$ such that $x^{j} \leq\left(\left(\mathcal{B} \oplus x^{1}\right) \ldots\right) \oplus x^{j-1}$ holds for all $1 \leq j \leq t$, we define $\mathcal{B} \oplus\left(x^{1}, \ldots, x^{t}\right):=\left(\left(\mathcal{B} \oplus x^{1}\right) \ldots\right) \oplus x^{t}$. Notice that the order of adding the vertices does make a difference, as it might be that some orderings are possible while some other orderings are not. The total number of added edges then equals $\sum_{j=1}^{t} \delta\left(x^{j}\right)$. 
Now, we can define the problem of anonymizing a general block sequence as follows:

General Block Sequence Anonymization (VA)

Input: $\quad$ A general block sequence $\mathcal{B}$ and $k, t, s \in \mathbb{N}$.

Question: Are there vertices $x^{1}, \ldots, x^{t^{\prime}}, t^{\prime} \leq t$, with $\sum_{j=1}^{t^{\prime}} \delta\left(x_{j}\right) \leq s$ such that $\mathcal{B}^{\prime}:=\mathcal{B} \oplus\left(x^{1}, \ldots, x^{t^{\prime}}\right)$ is $k$-anonymous, that is, either $b_{i}^{\prime} \geq k$ or $b_{i}^{\prime}=0$ holds for each $b_{i}^{\prime}$ in $\mathcal{B}^{\prime}$ ?

Note that this definition ensures that any Degree Anonymization (VA) instance $(G, k, t, s)$ is a yes-instance if and only if $\left(\left(\left|B_{0}\right|, \ldots,\left|B_{n-1}\right|\right), k, t, s\right)$ is a yes-instance of General Block Sequence Anonymization (VA). Therefore, in order to prove Theorem 10, it is sufficient to show that GENERAL BLOCK Sequence Anonymization (VA) is fixed-parameter tractable with respect to $s$. To this end, we give a kernelization algorithm.

Lemma 2. General Block Sequence Anonymization (VA) admits a problem kernel with respect to the maximum number s of newly inserted edges. The kernel is of size $s^{O(s)}$ and can be computed in linear time.

To prove Lemma 2, we will introduce several polynomial-time data reduction rules. We give an overview of these rules now. First, we upper-bound the anonymity level $k$ (Reduction Rule 1 ). Then, we upper-bound the maximum block size, that is $\max _{i}\left(b_{i}\right)$ (Reduction Rule 2). After that, we upper-bound the number $t$ of added edges (Reduction Rule 3), followed by upper-bounding the number of bad blocks, that is, the number of blocks that have strictly less than $k$ vertices, but also strictly more than 0 vertices (Reduction Rule 4). We then upper-bound the number of non-empty blocks (Reduction Rule 5), and finally, upper-bound the overall number of blocks (Reduction Rule 6). We mention here that all our reduction rules are easily seen to be polynomial-time computable, therefore, in what follows, we only explicitly prove their correctness.

We are ready to delve into the details. In order to prove the correctness of some of these reduction rules, we need the following simple lemma, which states that a sequence $\left(x^{1}, \ldots, x^{t}\right)$ of vertices that can be added to a general block sequence $\mathcal{B}$ can also be added to another general block sequence $\mathcal{B}^{\prime}$ of equal length if each entry in $\mathcal{B}^{\prime}$ has value of at least $\sum_{j=1}^{t} \delta\left(x^{j}\right)$.

Lemma 3. Let $\mathcal{B}=\left(b_{0}, \ldots, b_{d}\right)$ be a general block sequence and let $x^{1}, \ldots, x^{t}$ be a sequence of vertices such that $x^{j} \leq\left(\left(\mathcal{B} \oplus x^{1}\right) \ldots\right) \oplus x^{j-1}$ holds for all $1 \leq j \leq t$. Further, let $s:=\sum_{j=1}^{t} \delta\left(x^{j}\right)$ and let $\mathcal{B}^{\prime}=\left(b_{0}^{\prime}, \ldots, b_{d}^{\prime}\right)$ be a general block sequence with $b_{i}^{\prime} \geq \min \left\{b_{i}, s\right\}$ for all $i \in\{0, \ldots, d\}$.

Then, also $x^{j} \leq\left(\left(\mathcal{B}^{\prime} \oplus x^{1}\right) \ldots\right) \oplus x^{j-1}$ holds for all $1 \leq j \leq t$.

Proof. Let $I:=\left\{i \mid s \leq b_{i}^{\prime}<b_{i}\right\}$ be the set of indices where $\mathcal{B}^{\prime}$ is strictly less than $\mathcal{B}$ but at least $s$. Note that for all other indices $i$, we have $b_{i}^{\prime} \geq b_{i}$. Moreover, note that, for each $i \in\{0, \ldots, d\}$, it holds that $\sum_{j=1}^{t} x_{i}^{j} \leq s$, and thus, clearly, also $x_{i}^{j} \leq s-\sum_{l=1}^{j-1} x_{i}^{l}$ holds for each $j \in\{1, \ldots, t\}$. 
We prove the lemma by induction on $j$. For $j=1$, by assumption, we have $x_{i}^{1} \leq \min \left\{b_{i}, s\right\} \leq b_{i}^{\prime}$ for all $i \in\{0, \ldots, d\}$, and thus $x^{1} \leq \mathcal{B}^{\prime}$. Now, for $j \geq 2$, let $\mathcal{B}^{\prime}(j-1):=\mathcal{B}^{\prime} \oplus\left(x^{1}, \ldots, x^{j-1}\right)=\left(b_{0}^{\prime}(j-1), \ldots, b_{q}^{\prime}(j-1)\right)$ for some $q \in \mathbb{N}$. This is well-defined by the inductive hypothesis. Note that for all $i \in\{0, \ldots, q\} \backslash I$, we have $b_{i}^{\prime}(j-1) \geq b_{i}(j-1) \geq x_{i}^{j}$. For each $i \in I$, it holds $b_{i}^{\prime}(j-1) \geq s-\sum_{l=1}^{j-1} x_{i}^{l} \geq x_{i}^{j}$. Hence, $x^{j} \leq \mathcal{B}^{\prime}(j-1)$.

Let us now specify the reduction rules. The first reduction rule upper-bounds the degree $k$ of anonymity linearly in $s$.

Reduction Rule 1. We transform an instance $\left(\left(b_{0}, \ldots, b_{d}\right), k, t, s\right)$ of GENERAL Block Sequence Anonymization (VA) to the instance $\left(\left(b_{0}^{\prime}, \ldots, b_{d}^{\prime}\right), k^{\prime}, t, s\right)$, where $k^{\prime}:=\min \{k, 2 s+1\}$ and, for $i \in\{0, \ldots, d\}$, we set

$$
b_{i}^{\prime}:= \begin{cases}b_{i}-\left(k-k^{\prime}\right), & b_{i} \geq k-s \\ b_{i}, & \text { else }\end{cases}
$$

Lemma 4. Reduction Rule 1 is correct.

Proof. The general intuition behind this rule is that if $k$ is very large (with respect to $s$ ), then any small block must be somehow "fixed" (either by moving some vertices into it, or by moving all of the vertices in it to some other blocks). Therefore, for these blocks, the actual $k$ could be just slightly larger than their sizes. Moreover, for any large block, not all of the vertices of this block can be moved to another block. Therefore, $k$ and their sizes can be decreased.

More formally, we have to show that $I:=\left(\mathcal{B}=\left(b_{0}, \ldots, b_{d}\right), k, t, s\right)$ is a yesinstance if and only if $I^{\prime}:=\left(\mathcal{B}^{\prime}=\left(b_{0}^{\prime}, \ldots, b_{d}^{\prime}\right), k^{\prime}, t, s\right)$ is a yes-instance. Clearly, this is true for $k^{\prime}=k$ since this implies $I=I^{\prime}$. Thus, we can assume that $k^{\prime}=$ $2 s+1<k$. Therefore, $b_{i}^{\prime} \geq \min \left\{b_{i}, k-s-\left(k-k^{\prime}\right)\right\}=\min \left\{b_{i}, k^{\prime}-s\right\} \geq \min \left\{b_{i}, s\right\}$ holds for each $i \in\{0, \ldots, d\}$. Let $\left(x^{1}, \ldots, x^{t^{\prime}}\right)$, for $t^{\prime} \leq t$, be a sequence of vertices with $\sum_{j=1}^{t^{\prime}} \delta\left(x^{j}\right) \leq s$ such that

$$
\mathcal{B}\left(t^{\prime}\right):=\mathcal{B} \oplus\left(x^{1}, \ldots, x^{t^{\prime}}\right)=\left(b_{0}\left(t^{\prime}\right), \ldots, b_{q}\left(t^{\prime}\right)\right)
$$

is well-defined. Then, by Lemma 3, also

$$
\mathcal{B}^{\prime}\left(t^{\prime}\right):=\mathcal{B}^{\prime} \oplus\left(x^{1}, \ldots, x^{t^{\prime}}\right)=\left(b_{0}^{\prime}\left(t^{\prime}\right), \ldots, b_{q}^{\prime}\left(t^{\prime}\right)\right)
$$

is well-defined. Now, for $i \leq q$ such that $i>d$, we have $b_{i}^{\prime}\left(t^{\prime}\right)=b_{i}\left(t^{\prime}\right)$ since these values only depend on $\left(x^{1}, \ldots, x^{t^{\prime}}\right)$. Also, for all $i \leq d$ with $b_{i}=b_{i}^{\prime}$, it clearly holds that $b_{i}^{\prime}\left(t^{\prime}\right)=b_{i}\left(t^{\prime}\right)$. Finally, for the remaining indices $i$, we have $b_{i}\left(t^{\prime}\right)>0$ since $b_{i} \geq k-s>s+1$, and also $b_{i}^{\prime}\left(t^{\prime}\right)>0$ since $b_{i}^{\prime} \geq k-s-\left(k-k^{\prime}\right)=k^{\prime}-s=s+1$. Moreover, it holds

$$
\begin{aligned}
b_{i}^{\prime}\left(t^{\prime}\right)-b_{i}^{\prime} & =b_{i}\left(t^{\prime}\right)-b_{i} \quad \Leftrightarrow \\
b_{i}^{\prime}\left(t^{\prime}\right)-\left(b_{i}-\left(k-k^{\prime}\right)\right) & =b_{i}\left(t^{\prime}\right)-b_{i} \quad \Leftrightarrow \\
b_{i}^{\prime}\left(t^{\prime}\right)-k^{\prime} & =b_{i}\left(t^{\prime}\right)-k .
\end{aligned}
$$


Hence, $b_{i}^{\prime}\left(t^{\prime}\right) \geq k^{\prime}$ if and only if $b_{i}\left(t^{\prime}\right) \geq k$. Consequently, $\mathcal{B}\left(t^{\prime}\right)$ is $k$-anonymous if and only if $\mathcal{B}^{\prime}\left(t^{\prime}\right)$ is $k^{\prime}$-anonymous showing that $I$ is a yes-instance if and only if $I^{\prime}$ is a yes-instance.

The next reduction rule upper-bounds the maximum block size by $k+s$.

Reduction Rule 2. We transform an instance $\left(\left(b_{0}, \ldots, b_{d}\right), k, t, s\right)$ of GENERAL Block Sequence Anonymization (VA) to the instance $\left(\left(b_{0}^{\prime}, \ldots, b_{d}^{\prime}\right), k, t, s\right)$, where, for $i \in\{0, \ldots, d\}$, we set $b_{i}^{\prime}=\min \left\{k+s, b_{i}\right\}$.

Lemma 5. Reduction Rule 2 is correct.

Proof. The general idea of this rule is that the size of any very large block can be decreased. The basic reason is that we cannot use more than $s$ vertices of any block, including these large blocks.

More formally, let $I:=(\mathcal{B}, k, t, s)$ be an input instance and let $I^{\prime}:=$ $\left(\mathcal{B}^{\prime}, k, t, s\right)$ denote the transformed instance. Note that $b_{i}^{\prime} \geq \min \left\{b_{i}, s\right\}$ for each $i \in\{0, \ldots, d\}$. Let $\left(x^{1}, \ldots, x^{t^{\prime}}\right)$, for $t^{\prime} \leq t$, be a sequence of vertices with $\sum_{j=1}^{t^{\prime}} \delta\left(x^{j}\right) \leq s$ such that

$$
\mathcal{B}\left(t^{\prime}\right):=\mathcal{B} \oplus\left(x^{1}, \ldots, x^{t^{\prime}}\right)=\left(b_{0}\left(t^{\prime}\right), \ldots, b_{q}\left(t^{\prime}\right)\right)
$$

is well-defined. Then, again, by Lemma 3, also

$$
\mathcal{B}^{\prime}\left(t^{\prime}\right):=\mathcal{B}^{\prime} \oplus\left(x^{1}, \ldots, x^{t^{\prime}}\right)=\left(b_{0}^{\prime}\left(t^{\prime}\right), \ldots, b_{q}^{\prime}\left(t^{\prime}\right)\right)
$$

is well-defined. Clearly, for any $i$ with $b_{i}>k+s$, we have $b_{i}\left(t^{\prime}\right)>k$ and also $b_{i}^{\prime}\left(t^{\prime}\right) \geq k$ since $b_{i}^{\prime}=k+s$. For all other $i$, it holds $b_{i}^{\prime}\left(t^{\prime}\right)=b_{i}\left(t^{\prime}\right)$. Hence, $I$ is a yes-instance if and only if $I^{\prime}$ is a yes-instance.

Next, we upper-bound the number $t$ of new vertices in $s$ and $k$.

Reduction Rule 3. We transform an instance $(\mathcal{B}, k, t, s)$ of General Block Sequence Anonymization (VA) to the instance $(\mathcal{B}, k, \min \{t, k+2 s\}, s)$.

Lemma 6. Reduction Rule 3 is correct.

Proof. The general idea of this rule is that in any solution which uses a lot of new vertices, most of these new vertices must be isolated (that is, of degree 0 ). However, a solution does not need a lot of new isolated vertices, therefore, we can reduce $t$.

More formally, let $t^{*}:=\min \{t, k+2 s\}$. For $t^{*}=t$, there is nothing to show. Thus, assume that $t^{*}=k+2 s<t$. If $I:=(\mathcal{B}, k, t, s)$ is a no-instance, then, clearly, also $I^{\prime}:=\left(\mathcal{B}, k, t^{*}, s\right)$ is a no-instance since $t^{*}<t$. Thus, let $I$ be a yes-instance and let $t^{\prime}=t_{0}+t_{1} \leq t$ be the number of newly added vertices, where $t_{0}$ denotes the number of added degree-zero vertices and $t_{1}$ denotes the number of added vertices of degree at least one (by saying the degree of the vertex we mean, naturally, the total sum of the elements in the vertex vector). Let $\left(x^{1}, \ldots, x^{t^{\prime}}\right)$ be the added vertices with $\sum_{j=1}^{t^{\prime}} \delta\left(x^{j}\right) \leq s$ and note that we can 
assume that the $t_{0}$ first vertices $x^{1}=\ldots=x^{t_{0}}=(0, \ldots, 0)$ are the degree-zero vertices. Moreover, note that $t_{1} \leq s$ holds. Let $\mu:=\min \left\{t_{0}, k+s\right\}$. We show that adding the $\mu+t_{1} \leq k+s+s=t^{*}$ vertices $\left(x^{1}, \ldots, x^{\mu}, x^{t_{0}+1}, \ldots, x^{t^{\prime}}\right)$ is a solution for $I^{\prime}$. This is clearly true for $\mu=t_{0}$, hence we can assume that $\mu=$ $k+s<t_{0}$. First, we have to show that $\mathcal{B} \oplus\left(x^{1}, \ldots, x^{\mu}, x^{t_{0}+1}, \ldots, x^{t^{\prime}}\right)$ is welldefined. Trivially, $\mathcal{B} \oplus\left(x^{1}, \ldots, x^{\mu}\right)$ is well-defined. Let $\mathcal{B}(\mu):=\mathcal{B} \oplus\left(x^{1}, \ldots, x^{\mu}\right)$ and let $\mathcal{B}\left(t_{0}\right):=\mathcal{B} \oplus\left(x^{1}, \ldots, x^{t_{0}}\right)$. Note that we have $b_{0}(\mu)=b_{0}+\mu \geq s$ and, for all $i>0$, we have $b_{i}(\mu)=b_{i}\left(t_{0}\right)=b_{i}$. Thus, since by assumption $\mathcal{B}\left(t^{\prime}\right):=\mathcal{B}\left(t_{0}\right) \oplus\left(x^{t_{0}+1}, \ldots, x^{t^{\prime}}\right)$ is well-defined, it follows from Lemma 3 that also $\mathcal{B}^{*}:=\mathcal{B}(\mu) \oplus\left(x^{t_{0}+1}, \ldots, x^{t^{\prime}}\right)$ is well-defined. Note also that the total number of added edges does not change.

It remains to show that $\mathcal{B}^{*}$ is $k$-anonymous. This is true since $b_{i}^{*}=b_{i}\left(t^{\prime}\right)$ holds for each $i>0$, and $b_{0}^{*} \geq k$ holds since $b_{0}(\mu) \geq k+s$.

The next two reduction rules upper-bound the number of non-zero entries in the block sequence. First, we identify those blocks whose sizes need to be changed. Recall that a block is said to be bad if it contains less than $k$ but more than zero vertices.

The general idea for these two reduction rules is to keep all blocks of degree at most $2 s$ (since new vertices may end up in the first $s$ blocks and an already existing vertex with degree at most $s$ can reach degree at most $2 s$ ), the bad blocks (since we have to fix them), some good blocks close to the bad blocks (to allow movement of vertices to or from bad blocks), and also some further good blocks (to set the correct degrees of the newly added vertices).

Our next reduction rule transforms the instance into a trivial no-instance if there are more bad blocks than one could fix by adding at most $s$ edges. Each new edge introduced by a new vertex $x$ with $x_{i}>1$ can fix at most three bad blocks, namely $b_{i}, b_{i+1}$, and $b_{\delta(x)}$. Note that other block sizes are not affected by the edges corresponding to $x_{i}$.

Reduction Rule 4. Let $(\mathcal{B}, k, t, s)$ be an instance of GENERAL BloCK SEQUENCE ANONYMizATION (VA) with $\mathcal{B}=\left(b_{0}, \ldots, b_{d}\right)$. If $\mathcal{B}$ contains more than 3 s entries $b_{i}$ with $0<b_{i}<k$, then return a trivial no-instance.

Reduction Rule 4 is obviously correct.

In order to upper-bound the number of good blocks, the decisive observation is that adding two edges to good blocks cannot be considered to be independent. We first explain the intuition behind.

For example, adding an edge to a vertex from a degree- $i$ block of size $k$ may only be possible if one also adds an edge to a vertex from a degree- $(i-1)$ block - one vertex moves from the degree- $i$ block to the degree degree- $(i+1)$ block (causing it to be momentarily bad) and one moves from the degree- $(i-1)$ block to the degree- $i$ block and block $i$ ends up with size $k$.

The idea now is to consider consecutive blocks where operations on one block have influence on operations on the next block. Fortunately, the number of operations influencing each other is upper-bounded by the total number $s$ of added edges. 
More formally, we use the concepts of "scope" and "chain". Let $\mathcal{B}=$ $\left(b_{0}, \ldots, b_{d}\right)$ be a general block sequence. The scope of a position $z>s$ in $\mathcal{B}$ is the sequence of positions $(z-s, \ldots, z)$. The scope fingerprint $F_{z}$ of $z$ is the subsequence $F_{z}:=\left(b_{z-s}, \ldots, b_{z}\right)$. Let $\left(x^{1}, \ldots, x^{t}\right)$ be a sequence of vertices. A chain with respect to $\left(x^{1}, \ldots, x^{t}\right)$ is a pair $(y, z)$ of positive integers with $y<z$ such that $\forall i \in\{y, \ldots, z-1\}: \exists x^{j}: x_{i}^{j}>0$ (that is, every degree in the chain is moved). A chain $(y, z)$ is maximal if $(y-1, z)$ and $(y, z+1)$ are no chains.

Note that a chain has length at most $s$, that is, $z-y \leq s$, because the total number of added edges is at most $s$. That is, every (maximal) chain $(y, z)$ is "fully contained" in the scope of $z$.

We are now ready to formulate our most technical reduction rule which upper-bounds the number of good blocks which are not empty. It will iteratively mark positions in $\mathcal{B}$ corresponding to blocks that have to be kept and finally set the entries in $\mathcal{B}$ at all non-marked positions to zero.

Reduction Rule 5. Let $(\mathcal{B}, k, t, s)$ be an instance of General Block SEQUence Anonymization (VA) with $\mathcal{B}=\left(b_{0}, \ldots, b_{d}\right)$.

1. Mark the positions $0, \ldots, 2 s$.

2. Mark all positions $i$ with $\exists z:|z-i| \leq s \wedge 0<b_{z}<k$.

3. Iteratively do the following starting with $j:=0$.

(a) Find the next non-marked position $z>j$.

(b) Compute the scope fingerprint $F_{z}$ and set $j:=z$.

(c) If $F_{z}$ has been computed before less than $2 s^{2}+s$ times, then mark positions $z-s, \ldots, z$.

4. For each $0 \leq i \leq d$, set $b_{i}^{\prime}:=b_{i}$ if position $i$ is marked, and $b_{i}^{\prime}:=0$ otherwise.

$\operatorname{Return}\left(\mathcal{B}^{\prime}=\left(b_{0}^{\prime}, \ldots, b_{d}^{\prime}\right), k, t, s\right)$.

Lemma 7. Reduction Rule 5 is correct.

Proof. The general idea of this rule is that, besides the first $2 s$ blocks (which are important because the old vertices and the new vertices can reside in them at the end), and besides the chains corresponding to bad blocks, all other chains can be used only in order to let the new vertices achieve some desired degree. As we have only a limited number of such possible chains, and as they are used only by the new vertices, we can upper-bound the number of such useful chains.

More formally, first, assume that there is a solution $\left(\bar{x}^{1}, \ldots, \bar{x}^{t^{\prime}}\right), t^{\prime} \leq t$, such that $\mathcal{B}^{\prime} \oplus\left(\bar{x}^{1}, \ldots, \bar{x}^{t^{\prime}}\right)$ is $k$-anonymous. It is easy to verify that $\mathcal{B} \oplus\left(\bar{x}^{1}, \ldots, \bar{x}^{t^{\prime}}\right)$ is also $k$-anonymous.

Second, assume that there is a solution $\left(x^{1}, \ldots, x^{t^{\prime}}\right), t^{\prime} \leq t$, such that $\mathcal{B} \oplus\left(x^{1}, \ldots, x^{t^{\prime}}\right)$ is $k$-anonymous. We show how to adjust $\left(x^{1}, \ldots, x^{t^{\prime}}\right)$ to obtain a solution $\left(\bar{x}^{1}, \ldots, \bar{x}^{t^{\prime}}\right)$ such that $\mathcal{B}^{\prime} \oplus\left(\bar{x}^{1}, \ldots, \bar{x}^{t^{\prime}}\right)$ is $k$-anonymous. Intuitively, we 
show that every maximal chain $(y, z)$ with respect to $\left(x^{1}, \ldots, x^{t^{\prime}}\right)$ can either be realized equivalently with respect to $\left(\bar{x}^{1}, \ldots, \bar{x}^{t^{\prime}}\right)$ or else we show a replacement ensuring $k$-anonymity.

We construct $\left(\bar{x}^{1}, \ldots, \bar{x}^{t^{\prime}}\right)$ as follows. First, we initialize $\bar{x}^{j}=(0, \ldots, 0)$ of the same length as $x^{j}$ for each $1 \leq j \leq t^{\prime}$. For each maximal chain $(y, z)$ with respect to $\left(x^{1}, \ldots, x^{t^{\prime}}\right)$, we distinguish two cases:

Case 1. $\forall i \in\{y, \ldots, z\}: b_{i}=b_{i}^{\prime}$. We set $\bar{x}_{i}^{j}:=x_{i}^{j}$ for all $j \in\left\{1, \ldots, t^{\prime}\right\}$, $i \in\{y, \ldots, z\}$, that is, this chain can be realized equivalently.

Case 2. $\exists i \in\{y, \ldots, z\}: b_{i} \geq k$ but $b_{i}^{\prime}=0$. This is only possible because not all positions between $y$ and $z$ have been marked by the reduction rule. Hence, the scope fingerprint $F_{z}$ of $z$ must have been computed more than $2 s^{2}+s$ times before.

In the following, we say that the scope of $z$ is touched by a sequence of vertices $\left(x^{1}, \ldots, x^{t^{\prime}}\right)$ if $\exists j \in\left\{1, \ldots, t^{\prime}\right\}: \exists i \in\{z-s, \ldots, z\}: x_{i}^{j}>0$.

We show that there is at least one position $z^{\prime}$ with fingerprint $F_{z^{\prime}}=F_{z}$ whose scope is neither touched by $\left(x^{1}, \ldots, x^{t^{\prime}}\right)$ nor by $\left(\bar{x}^{1}, \ldots, \bar{x}^{t^{\prime}}\right)$ as constructed so far. Note that each edge touches at most $s+1$ scopes (as each scope is basically an interval of size $s$ ). Moreover, there are at most $s$ edges in $\left(x^{1}, \ldots, x^{t}\right)$ and, until now, at most $s-1$ (possibly different) edges in $\left(\bar{x}^{1}, \ldots, \bar{x}^{t}\right)$ have been introduced. Altogether, at most $(2 s-1) \cdot(s+1)=2 s^{2}+s-1$ scopes (with any fingerprint) have been touched. In particular, there is one scope $\left(z^{\prime}-s, \ldots, z^{\prime}\right)$ with scope fingerprint $F_{z}$ that is not touched so far by $\left(\bar{x}^{1}, \ldots, \bar{x}^{t}\right)$ and that is also not touched by $\left(x^{1}, \ldots, x^{t}\right)$.

Finally, we use the scope of $z^{\prime}$ to realize $(y, z)$ by setting $\bar{x}_{i-z+z^{\prime}}^{j}:=x_{i}^{j}$ for all $j \in\left\{1, \ldots, t^{\prime}\right\}, i \in\{y, \ldots, z\}$.

By construction of $\left(\bar{x}^{1}, \ldots, \bar{x}^{t^{\prime}}\right)$, the number of newly added edges is

$$
\sum_{j=1}^{t^{\prime}} \delta\left(\bar{x}^{j}\right)=\sum_{j=1}^{t^{\prime}} \delta\left(x^{j}\right) .
$$

More precisely, it even holds that the degrees of the newly introduced vertices remain unchanged, that is,

$$
\forall j \in\{1, \ldots, t\}: \delta\left(\bar{x}^{j}\right)=\delta\left(x^{j}\right) .
$$

Now, let $\hat{\mathcal{B}}:=\left(\hat{b}_{0}, \ldots, \hat{b}_{d}\right)=\mathcal{B}^{\prime} \oplus\left(\bar{x}^{1}, \ldots, \bar{x}^{t^{\prime}}\right)$ and $\check{\mathcal{B}}:=\left(\check{b}_{0}, \ldots, \check{b}_{d}\right)=$ $\mathcal{B} \oplus\left(x^{1}, \ldots, x^{t^{\prime}}\right)$. It remains to show that $\hat{\mathcal{B}}$ is $k$-anonymous. To this end, consider an arbitrary index $h$ in $\hat{\mathcal{B}}$.

Case 1. $h$ is neither in a maximal chain with respect to $\left(x^{1}, \ldots, x^{t^{\prime}}\right)$ nor with respect to $\left(\bar{x}^{1}, \ldots, \bar{x}^{t^{\prime}}\right)$. By construction of $\left(\bar{x}^{1}, \ldots, \bar{x}^{t^{\prime}}\right)$, this solution introduces the same number of new vertices with degree $h$ as the solution $\left(x^{1}, \ldots, x^{t^{\prime}}\right)$ does. Thus, $\hat{b}_{h}=\check{b_{h}}$ and $\left(\check{b}_{h}=0\right) \vee\left(\check{b}_{h} \geq k\right)$. 
Case 2. $h$ is in a maximal chain $(y, z)$ with respect to $\left(x^{1}, \ldots, x^{t^{\prime}}\right)$, and in a maximal chain $\left(y^{\prime}, z^{\prime}\right)$ with respect to $\left(\bar{x}^{1}, \ldots, \bar{x}^{\prime}\right)$. By construction of $\left(\bar{x}^{1}, \ldots, \bar{x}^{t^{\prime}}\right)$, it holds that $y^{\prime}=y, z^{\prime}=z$, and $\forall j \in\left\{1, \ldots, t^{\prime}\right\}: \forall i \in$ $\{y, \ldots, z\}: \bar{x}_{i}^{j}:=x_{i}^{j}$. Furthermore, $\left(\bar{x}^{1}, \ldots, \bar{x}^{t^{\prime}}\right)$ introduces the same number of new vertices with degree $h$ as $\left(x^{1}, \ldots, x^{t^{\prime}}\right)$ does. Hence, $\hat{b}_{h}=\check{b_{h}}$ and $\left(\check{b}_{h}=0\right) \vee\left(\check{b}_{h} \geq k\right)$.

Case 3. $h$ is in a maximal chain $(y, z)$ with respect to $\left(x^{1}, \ldots, x^{t^{\prime}}\right)$, but not in a maximal chain with respect to $\left(\bar{x}^{1}, \ldots, \bar{x}^{t^{\prime}}\right)$. We claim that $\delta\left(x^{j}\right) \neq h$ (and thus, also $\delta\left(\bar{x}^{j}\right) \neq h$ ) holds for all $j \in\left\{1 \ldots, t^{\prime}\right\}$, and prove this as follows. Assume towards a contradiction that there is a new vertex $x^{j}$ with degree $\delta\left(x^{j}\right)=h$. Then, $h \leq s$. However, all positions up to $2 s$ have been marked in Step 1 of the reduction rule. In particular, all positions between $y$ and $z$ have been marked and, hence, by construction of $\left(\bar{x}^{1}, \ldots, \bar{x}^{t^{\prime}}\right)$ the pair $(y, z)$ would also be a maximal chain with respect to $\left(\bar{x}^{1}, \ldots, \bar{x}^{t^{\prime}}\right)$. This contradicts the assumption of Case 3, and hence $z>2 s$ and $h>s$, and there is no new vertex with degree $h$. Analogously, $h$ does not correspond to a bad block because then all positions between $y$ and $z$ would have been marked in Step 2 of the reduction. Thus, $\hat{b}_{h}=b_{h}^{\prime}$ and $\left(b_{h}^{\prime}=0\right) \vee\left(b_{h}^{\prime} \geq k\right)$.

Case 4. $h$ is not in a maximal chain with respect to $\left(x^{1}, \ldots, x^{t^{\prime}}\right)$, but in a maximal chain $(\bar{y}, \bar{z})$ with respect to $\left(\bar{x}^{1}, \ldots, \bar{x}^{t^{\prime}}\right)$. This is only possible if there is a position $z$ with the same scope fingerprint as $z^{\prime}$ such that $(z-(\bar{z}-\bar{y}), z)$ is a maximal chain with respect to $\left(x^{1}, \ldots, x^{t^{\prime}}\right)$ and

$$
\forall j \in\left\{1, \ldots, t^{\prime}\right\}: \forall i \in\{\bar{y}, \ldots, \bar{z}\}: \bar{x}_{i}^{j}:=x_{i-\bar{z}+z}^{j} .
$$

Furthermore, neither $\left(x^{1}, \ldots, x^{t^{\prime}}\right)$ nor $\left(\bar{x}^{1}, \ldots, \bar{x}^{t^{\prime}}\right)$ introduce new vertices with degree $h$ because all positions up to $2 s$ have been marked in Step 1 of the reduction, and hence, $z>2 s$. That is, $\hat{b}_{h}=\breve{b}_{h-\bar{z}+z}$ and $\left(\breve{b}_{h-\bar{z}+z}=\right.$ $0) \vee\left(\check{b}_{h-\bar{z}+z} \geq k\right)$.

Hence, $\hat{\mathcal{B}}$ is indeed $k$-anonymous.

It remains to upper-bound the largest degree by some function in $s$. To this end, observe the following with respect to high-degree blocks. First, by adding at most $s$ edges, no new vertex can end up in a block of vertices with degree larger than $s$. Second, by adding some vertices and at most $s$ edges, we cannot decrease the degree of any original vertex and we can only increase the degree of an original vertex by at most $s$.

Based on the observations above, we introduce the concept of "high-degree large gaps" as follows. Let $\left(\mathcal{B}=\left(b_{0}, \ldots, b_{d}\right), k, t, s\right)$ be an instance of GENERAL Block Sequence Anonymization (VA). We say that a pair of positive integers $(\ell, r)$ describes a high-degree large gap of $\mathcal{B}$ if

- $s<\ell<r$,

- $r-\ell>s$, and 
- $\forall i \in\{\ell, \ldots, r\}: b_{i}=0$.

Our final reduction rule shrinks the high-degree large gaps in a general block sequence.

Reduction Rule 6. We transform an instance $(\mathcal{B}, k, t, s)$ of General Block Sequence AnOnymization (VA) with some high-degree large gap $(\ell, r)$ in $\mathcal{B}$, to the instance $\left(\mathcal{B}^{\prime}, k, t, s\right)$, where $\mathcal{B}^{\prime}$ is constructed from $\mathcal{B}$ by removing the entries $b_{\ell+s}, \ldots, b_{r}$.

Lemma 8. Reduction Rule 6 is correct.

Proof. The general idea of this rule is that the size of any such high-degree large gap can be reduced, as no vertex can cross-over this gap.

More formally, we first observe that the entries $b_{\ell+s}, \ldots, b_{r}$ are all 0 -entries in $\mathcal{B}$. Moreover, in any $\mathcal{B}^{j}:=\mathcal{B} \oplus\left(x^{1}, \ldots, x^{j}\right)$, inserting at most $s$ edges to $\mathcal{B}$, these entries $b_{\ell+s}, \ldots, b_{r}$ are all 0 -entries.

First, assume that there is a solution $\left(x^{1}, \ldots, x^{t^{\prime}}\right), t^{\prime} \leq t$, such that $\mathcal{B} \oplus$ $\left(x^{1}, \ldots, x^{t^{\prime}}\right)$ is $k$-anonymous. Then, obtain $\bar{x}^{j}$ from $x^{j}$ by removing the entries $x_{\ell+s}^{j}, \ldots, x_{r}^{j}$ for each $1 \leq j \leq t^{\prime}$. (Note that these entries must be 0 -entries.) It is easy to verify that $\mathcal{B}^{\prime} \oplus\left(\bar{x}^{1}, \ldots, \bar{x}^{t^{\prime}}\right)$ is $k$-anonymous.

Second, assume that there is a solution $\left(\bar{x}^{1}, \ldots, \bar{x}^{t^{\prime}}\right)$ such that $\mathcal{B}^{\prime} \oplus\left(\bar{x}^{1}, \ldots, \bar{x}^{t^{\prime}}\right)$ is $k$-anonymous. Then, obtain $x^{j}$ from $\bar{x}^{j}$ by inserting $r-\ell-s 0$-entries between $\bar{x}_{\ell-1}^{j}$ and $\bar{x}_{\ell}^{j}$. It is easy to verify that $\mathcal{B} \oplus\left(x^{1}, \ldots, x^{t^{\prime}}\right)$ is $k$-anonymous.

For proving Lemma 2, it remains to show that the above reduction rules indeed yield a problem kernel with respect to $s$.

Proof (of Lemma 2). Let $I:=(\mathcal{B}, k, t, s)$ be an arbitrary General Block Sequence Anonymization (VA) instance. Our kernelization algorithm first applies to $I$ Reduction Rules 1,2 , and 3 , in that order. Let $I^{\prime}:=\left(\mathcal{B}^{\prime}, k^{\prime}, t^{\prime}, s\right)$ be the instance achieved after that. Clearly, we have $k^{\prime} \in O(s), \max _{i} b_{i}^{\prime} \leq$ $k^{\prime}+s \in O(s)$, and also $t^{\prime} \leq k^{\prime}+2 s \in O(s)$. Thus, all numbers in $I^{\prime}$ are upper-bounded by $s$. In order to upper-bound the maximum degree in $s$, we apply Reduction Rules 4 and 5 once, and Reduction Rule 6 exhaustively to $I^{\prime}$. We denote the resulting instance by $I^{\prime \prime}:=\left(\mathcal{B}^{\prime \prime}, k^{\prime}, t^{\prime}, s\right)$. After application of Reduction Rule $4, I^{\prime \prime}$ either is a constant-size no-instance, or $\mathcal{B}^{\prime \prime}$ contains at most $3 s$ bad blocks. Now, consider the number of marked positions in each step of Reduction Rule 5. In Step 1, we mark $2 s+1$ positions. Step 2 marks at most $3 s(2 s+1)$ positions, whereas in Step 3 we mark at most $\left(2 s^{2}+s\right) s$ positions for each possible scope fingerprint. The number of possible scope fingerprints is $\left(\max _{i} b_{i}^{\prime}+1\right)^{s} \in s^{O(s)}$. Thus, the total number of non-zero blocks in $\mathcal{B}^{\prime \prime}$ is bounded by a function in $s$. Finally, after exhaustive application of Reduction Rule 6 there are no more large-degree gaps in $\mathcal{B}^{\prime \prime}$, hence, the number of degree-zero blocks in $\mathcal{B}^{\prime \prime}$ is at most $s$ times the number of non-zero blocks, which is again bounded in $s$. The correctness is guaranteed by Lemmas 4 to 8 . Clearly, this process can be done in linear time since each reduction rule is applied at most a linear number of times and runs in linear time. The size of the 
kernel is governed by the number of possible chains, therefore upper-bounded by $s^{O(s)}$. Finally, since each reduction rule can be carried in polynomial time, it follows that the kernelization can be performed in polynomial time.

\section{Conclusion}

Table 1 in the introductory section overviews most of our results and leaves several specific open questions. Moreover, it is fair to say that our positive algorithmic results are basically of classification nature only and require further improvement for practical relevance. Indeed, a more holistic approach in terms of a full-fledged multivariate complexity analysis [15, 30], perhaps also driven by the analysis of real-world network data characteristics, may help to derive practically useful algorithmic results. A deeper investigation of approximation algorithms (cf. [8, 9]) may be beneficial as well. Finally, typical social network properties such as measured by the clustering coefficient or the average path length are studied in experimental work [8], but the computational complexity of I-PREserving Degree AnONymization (VA) with respect to these properties is unexplored so far.

\section{Bibliography}

[1] N. Alon, G. Gutin, E. J. Kim, S. Szeider, and A. Yeo. Solving MAX-r-SAT above a tight lower bound. Algorithmica, 61(3):638-655, 2011. (cited on 3)

[2] C. Bazgan and A. Nichterlein. Parameterized inapproximability of degree anonymization. In Proceedings of the 9th International Symposium on Parameterized and Exact Computation (IPEC '14), volume 8894 of LNCS. Springer, 2014. (cited on 2)

[3] L. Bilge, T. Strufe, D. Balzarotti, and E. Kirda. All your contacts are belong to us: automated identity theft attacks on social networks. In Proceedings of the 18th International Conference on World Wide Web (WWW'09), pages 551-560. ACM, 2009. (cited on 3)

[4] R. Bredereck, S. Hartung, A. Nichterlein, and G. J. Woeginger. The complexity of finding a large subgraph under anonymity constraints. In Proceedings of the 24th International Symposium on Algorithms and Computation (ISAAC '13), volume 8283 of $L N C S$, pages 152-162. Springer, 2013. (cited on 2)

[5] R. Bredereck, V. Froese, S. Hartung, A. Nichterlein, R. Niedermeier, and N. Talmon. The complexity of degree anonymization by vertex addition. In Proceedings of the 10th International Conference on Algorithmic Aspects in Information and Management (AAIM '14), volume 8546 of Lecture Notes in Computer Science, pages 44-55. Springer, 2014. (cited on 1) 
[6] L. Cai, J. Chen, R. G. Downey, and M. R. Fellows. Advice classes of parameterized tractability. Annals of Pure and Applied Logic, 84(1):119138, 1997. (cited on 6)

[7] J. Casas-Roma, J. Herrera-Joancomartí, and V. Torra. An algorithm for $k$ degree anonymity on large networks. In Proceedings of the 2013 IEEE/ACM International Conference on Advances in Social Networks Analysis and Mining (ASONAM '13), pages 671-675. ACM Press, 2013. (cited on 2)

[8] S. Chester, B. M. Kapron, G. Ramesh, G. Srivastava, A. Thomo, and S. Venkatesh. Why Waldo befriended the dummy? $k$-anonymization of social networks with pseudo-nodes. Social Network Analysis and Mining, 3 (3):381-399, 2013. (cited on 2, 3, 31)

[9] S. Chester, B. M. Kapron, G. Srivastava, and S. Venkatesh. Complexity of social network anonymization. Social Network Analysis and Mining, 3(2): 151-166, 2013. (cited on 2, 31)

[10] K. L. Clarkson, K. Liu, and E. Terzi. Towards identity anonymization in social networks. In Link Mining: Models, Algorithms, and Applications, pages 359-385. Springer, 2010. (cited on 2)

[11] R. G. Downey and M. R. Fellows. Fundamentals of Parameterized Complexity. Springer, 2013. (cited on 6,8)

[12] E. Elkind, P. Faliszewski, and A. Slinko. Clone structures in voters' preferences. In Proceedings of the 13th ACM conference on electronic commerce (EC '12), pages 496-513. ACM, 2012. (cited on 3)

[13] P. Erdős and P. Kelly. The minimal regular graph containing a given graph. The American Mathematical Monthly, 70:1074-1075, 1963. (cited on 12)

[14] P. Erdős and T. Gallai. Graphs with prescribed degrees of vertices (in Hungarian). Matematikai Lapok, 11:264-274, 1960. (cited on 12)

[15] M. R. Fellows, B. M. P. Jansen, and F. A. Rosamond. Towards fully multivariate algorithmics: Parameter ecology and the deconstruction of computational complexity. European Journal of Combinatorics, 34(3):541566, 2013. (cited on 31)

[16] J. Flum and M. Grohe. Parameterized Complexity Theory. Springer, 2006. (cited on 6)

[17] M. R. Garey and D. S. Johnson. Computers and Intractability: A Guide to the Theory of NP-Completeness. Freeman, 1979. (cited on 7)

[18] J. Guo and R. Niedermeier. Invitation to data reduction and problem kernelization. ACM SIGACT News, 38(1):31-45, 2007. (cited on 6) 
[19] S. L. Hakimi. On realizability of a set of integers as degrees of the vertices of a linear graph. i. Journal of the Society for Industrial 85 Applied Mathematics, 10(3):496-506, 1962. (cited on 12)

[20] S. Hartung and N. Talmon. The complexity of degree anonymization by graph contractions. In Theory and Applications of Models of Computation, volume 9076 of $L N C S$, pages 260-271. Springer, 2015. (cited on 2)

[21] S. Hartung, C. Hoffmann, and A. Nichterlein. Improved upper and lower bound heuristics for degree anonymization in social networks. In Proceedings of the 13th International Symposium on Experimental Algorithms (SEA '14), volume 8504 of $L N C S$, pages 376-387. Springer, 2014. (cited on 2)

[22] S. Hartung, A. Nichterlein, R. Niedermeier, and O. Suchỳ. A refined complexity analysis of degree anonymization in graphs. Information and Computation, 2014. (cited on 2)

[23] V. Havel. A remark on the existence of finite graphs (in Czech). Ćasopis pro Pěstování Matematiky, 80(477-480):1253, 1955. (cited on 12)

[24] S. Kratsch. Recent developments in kernelization: A survey. Bulletin of the EATCS, 113:58-97, 2014. (cited on 3, 6)

[25] H. W. Lenstra. Integer programming with a fixed number of variables. Mathematics of Operations Research, 8:538-548, 1983. (cited on 19, 21)

[26] K. Liu and E. Terzi. Towards identity anonymization on graphs. In ACM SIGMOD International Conference on Management of Data (SIGMOD '08), pages 93-106. ACM, 2008. (cited on 1, 2, 17)

[27] X. Lu, Y. Song, and S. Bressan. Fast identity anonymization on graphs. In Proceedings of Database and Expert Systems Applications (DEXA '12), volume 7446 of $L N C S$, pages 281-295. Springer, 2012. (cited on 2)

[28] G. S. Lueker. Two NP-complete problems in nonnegative integer programming. Technical report, Computer Science Laboratory, Princeton University, 1975. (cited on 14)

[29] R. Niedermeier. Invitation to Fixed-Parameter Algorithms. Oxford University Press, 2006. (cited on 6)

[30] R. Niedermeier. Reflections on multivariate algorithmics and problem parameterization. In Proceedings of the 27th International Symposium on Theoretical Aspects of Computer Science (STACS '10), volume 5 of LIPIcs, pages 17-32. Schloss Dagstuhl-Leibniz-Zentrum für Informatik, 2010. (cited on 31 )

[31] B. Zhou and J. Pei. The $k$-anonymity and $l$-diversity approaches for privacy preservation in social networks against neighborhood attacks. Knowledge and Information Systems, 28(1):47-77, 2011. (cited on 2) 